\title{
The Role of Arabidopsis ABCG9 and ABCG31 ATP Binding Cassette Transporters in Pollen Fitness and the Deposition of Steryl Glycosides on the Pollen Coat ${ }^{\mathbb{W}}$
}

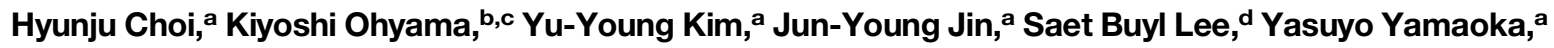 \\ Toshiya Muranaka, ${ }^{b, e}$ Mi Chung Suh, ${ }^{d}$ Shozo Fujioka, ${ }^{f}$ and Youngsook Lee ${ }^{a, 1}$ \\ a Pohang University of Science and Technology-University of Zurich Cooperative Laboratory, Department of Integrative Bioscience \\ and Biotechnology, Pohang University of Science and Technology, Pohang 790-784, Korea \\ ${ }^{b}$ RIKEN Center for Sustainable Resource Science, Tsurumi-ku, Yokohama, Kanagawa 244-0045, Japan \\ c Department of Chemistry and Materials Science, Graduate School of Science and Engineering, Tokyo Institute of Technology, \\ Meguro-ku, Tokyo 152-8551, Japan \\ d Department of Bioenergy Science and Technology, College of Agriculture and Life Sciences, Chonnam National University, Gwangju \\ 500-757, Korea \\ e Department of Biotechnology, Graduate School of Engineering, Osaka University, Suita-shi, Osaka 565-0871, Japan \\ ${ }^{f}$ RIKEN Advanced Science Institute, Wako-shi, Saitama 351-0198, Japan
}

The pollen coat protects pollen grains from harmful environmental stresses such as drought and cold. Many compounds in the pollen coat are synthesized in the tapetum. However, the pathway by which they are transferred to the pollen surface remains obscure. We found that two Arabidopsis thaliana ATP binding cassette transporters, ABCG9 and ABCG31, were highly expressed in the tapetum and are involved in pollen coat deposition. Upon exposure to dry air, many abcg9 abcg31 pollen grains

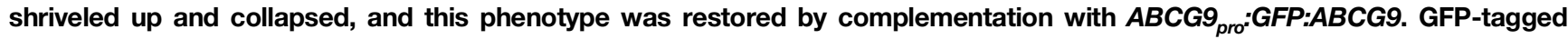
ABCG9 or ABCG31 localized to the plasma membrane. Electron microscopy revealed that the mutant pollen coat resembled the immature coat of the wild type, which contained many electron-lucent structures. Steryl glycosides were reduced to about half of wild-type levels in the abcg9 abcg31 pollen, but no differences in free sterols or steryl esters were observed. A mutant deficient in steryl glycoside biosynthesis, ugt80A2 ugt80B1, exhibited a similar phenotype. Together, these results indicate that steryl glycosides are critical for pollen fitness, by supporting pollen coat maturation, and that ABCG9 and ABCG31 contribute to the accumulation of this sterol on the surface of pollen.

\section{INTRODUCTION}

Pollen viability is one of the most important factors in determining seed yield. In Arabidopsis thaliana, pollen viability peaks just after anther dehiscence and remains high until the flower opens. Soon afterward, viability decreases drastically and is completely lost within $2 \mathrm{~d}$ (Pickert, 1988). Pollen viability can be affected by various environmental factors, such as temperature, humidity, and light (Rao et al., 1992; Lansac et al., 1994; Demchik and Day, 1996; Lee and Lee, 2003). The release of pollen from the anther often exposes the pollen to unfavorable conditions; thus, it is crucial that pollen is protected. Unlike other plant cells, which produce a classical cellulosic cell wall, pollen forms a pollen wall and coat. This compact pollen surface protects the pollen from various stresses, such as cold, heat, drought, and UV light shock (Pacini and Franchi, 1993).

Many recent reports revealed the mechanism of biosynthesis and transport of pollen wall precursors in Arabidopsis and showed

\footnotetext{
${ }^{1}$ Address correspondence to ylee@postech.ac.kr.

The author responsible for distribution of materials integral to the findings presented in this article in accordance with the policy described in the Instructions for Authors (www.plantcell.org) is: Youngsook Lee (ylee@ postech.ac.kr).

एOnline version contains Web-only data.

www.plantcell.org/cgi/doi/10.1105/tpc.113.118935
}

that the pollen wall is important for pollen development and protection (van der Meer et al., 1992; Matsuda et al., 1996; Aarts et al., 1997; Morant et al., 2007; de Azevedo Souza et al., 2009; Dobritsa et al., 2009, 2010; Quilichini et al., 2010; Xu et al., 2010; Choi et al., 2011; Dou et al., 2011). By contrast, studies on pollen coat lipids were mostly centered on pollen-stigma interactions, such as the self-incompatibility response and pollen hydration (Preuss et al., 1993; Piffanelli et al., 1998). However, it is becoming evident that the pollen coat is also involved in maintaining pollen viability under harsh environmental conditions, as can be deduced from the sensitivity of many pollen coatless mutants to arid conditions (Preuss et al., 1993; Jessen et al., 2011) and the hypersensitivity to UV light stress of several mutants with pollen deficient in flavonoids, a component of the pollen coat (Hsieh and Huang, 2007).

The pollen wall is composed of intine, which consists mainly of cellulose, and exine, which consists of sporopollenin, polymers of fatty acid derivatives, and phenylpropanoids. The pollen coat forms the outermost layer and is mainly composed of steryl esters but also contains small amounts of flavonoids and alkanes (Hsieh and Huang, 2007). Most of the materials that constitute the pollen wall and coat are supplied by the tapetum, whereas the intine is generated by the pollen grain itself. The tapetum, the innermost layer of the pollen sac, nourishes microspores throughout their development into mature pollen grains (Goldberg et al., 1993). The tapetum secretes glucanases, which separate tetrads into individual 
microspores (Stieglitz, 1977), and then secretes pollen exine precursors onto the pollen surface, thus contributing to pollen wall formation (Piffanelli et al., 1998; Scott et al., 2004). In the final stage of pollen development, the tapetum provides the pollen with materials for pollen coat formation. Tapetal cells accumulate lipidic molecules and proteins in suborganelles, such as endoplasmic reticulum-derived tapetosomes and plastid-derived elaioplasts. These suborganelles are released upon degradation of the tapetum, and their contents are deposited in the cavities between neighboring pollen exine baculae, thus completing pollen coat formation (Hsieh and Huang, 2005). However, it is not clear whether all of the materials that constitute the pollen coat are released in a chaotic manner during tapetum degradation or some are released by transporters in a more controlled manner prior to tapetum degradation.

If tapetal cells secrete some lipidic pollen coat precursors via a transporter, the most likely candidate transporter is the ATP binding cassette $(A B C)$ transporter. In many organisms, including animals, insects, fungi, and plants, ABCG subfamily members are reported to be involved in lipid transport ( $\mathrm{Li}$ and Prinz, 2004; Velamakanni et al., 2007; Kang et al., 2011), and the deletion of some members of the transporter family results in lipid-related diseases, growth defects, and sterility (Berge et al., 2000; Bird et al., 2007; Luo et al., 2007; Panikashvili et al., 2007; Ukitsu et al., 2007; Quilichini et al., 2010; Xu et al., 2010; Choi et al., 2011; Dou et al., 2011). Plants are highly enriched in a number of $A B C G$ transporters, but only several of these have been studied to date. $\mathrm{ABC}$ transporters often assume the role of transporting important molecules in specific directions, since they use ATP hydrolysis to energize the transport, and they are often polarly localized at a specific side of the cell (Geisler et al., 2005; Terasaka et al., 2005; Panikashvili et al., 2007; Strader and Bartel, 2009; Ruzicka et al., 2010; Bessire et al., 2011). Thus, this group of transporters would be an ideal candidate for the controlled secretion of coat materials to the developing male gametophyte.

Here, we report that two Arabidopsis ABC transporters, ABCG9 and $A B C G 31$, are highly expressed in the tapetum and that the corresponding double knockout mutant exhibits anther-specific phenotypes that were not observed in either single knockout mutant. Extensive phenotypic observations and lipid analysis revealed that the pollen of the double knockout mutant had an immature pollen coat, increased sensitivity to stress, and altered steryl glycoside content. Furthermore, the two transporters were predominantly expressed in tapetal cells and localized to the plasma membrane. Thus, this study suggests that these two $A B C$ transporters are involved in the deposition of steryl glycoside or related compounds, a process that is poorly understood in plants.

\section{RESULTS}

\section{$A B C G 9$ and $A B C G 31$ Are Highly Expressed in Male Reproductive Organs}

To identify candidate transporters that are involved in the transport of lipids to the pollen surface, we searched for Arabidopsis ABCG transporter genes that are highly expressed in a developing bud using an in silico database (the BAR Arabidopsis eFP browser [http://bar.utoronto.ca/efp/cgi-bin/efpWeb.cgi];
Supplemental Figure 1). Among the candidates, ABCG9 and $A B C G 31$ were specifically expressed in the stamen and in no other parts of developing flowers (Arabidopsis MPSS plus [http:// mpss.udel.edu/at/mpss_index.php]; Supplemental Data Set 1). Furthermore, no mRNA signal was detected for these genes in apetala3 or agamous1, which lack stamens (Bowman et al., 1989), but a strong signal was detected in sup ap1, which produces supernumerary stamens (Sakai et al., 2000). Interestingly, these two genes were highly coexpressed (Arabidopsis Coexpression Mining Tools [Manfield et al., 2006] and ATTEDII [http://atted.jp/top_search. shtml\#CoExSearch]). Moreover, no other ABC genes were found to be strongly coexpressed with either ABCG9 or ABCG31; ABCG31 was the only $A B C$ protein gene among the 150 genes identified as the most highly coexpressed genes with $A B C G 9$, and vice versa (Supplemental Data Set 2).

\section{The Pollen Grains of abcg9-1 abcg31-1 Plants Shrivel Up and Stick Together When Exposed to Dry Air}

For the functional analysis of $A B C G 9$ and $A B C G 31$ in planta, we obtained two independent $A B C G 9$ T-DNA insertional mutants (abcg9-1 and abcg9-2) from the SALK Institute and two independent $A B C G 31$ T-DNA insertional mutants from the SALK Institute (abcg31-1) and GABI-Kat (abcg31-2). abcg9-1 and abcg9-2 contained T-DNAs in the third and second exons of ABCG9, respectively (Supplemental Figure 2A), whereas abcg31-1 and abcg31-2 contained T-DNAs in the 19th intron and 10th exon of $A B C$ G31, respectively (Supplemental Figure 3A). The insertion sites were verified by genomic DNA-PCR (Supplemental Figures 2B and 3B). RT-PCR analysis confirmed that all of the homozygous mutant plants were complete knockouts (Supplemental Figures 2C and 3C).

Neither of the knockout plants displayed any visible defects in overall development. If $A B C G 31$ and $A B C G 9$ have similar functions, deletion of both $A B C G 31$ and $A B C G 9$ would lead to a more pronounced phenotype than that observed for either of the single knockout plants. Thus, we generated abcg9 abcg31 double knockout plants, abcg9-1 abcg31-1, by crossing abcg9-1 with abcg31-1. The homozygous knockout plants were identified using genomic DNA-PCR and RT-PCR (Supplemental Figures 4A and 4B, respectively). The double knockout plants exhibited no prominent aberrant phenotype in terms of gross morphology or development when grown under normal conditions.

To test whether the pollen surface of abcg9-1 abcg31-1 double knockout plants was defective, we observed wild-type and mutant pollen grains by scanning electron microscopy without any fixation step. In contrast to the wild-type and single knockout pollen, many of the abcg9-1 abcg31-1 pollen grains were shriveled up and clumped together (Figure 1, abcg9-1 abcg31-1). This phenotype was rescued by introducing $A B C G 9_{\text {pro }}$ :sGFP:ABCG9 into abcg9-1 abcg31-1 mutant plants (Figure 1, Compl-1). There was no difference between the genotypes in terms of reticular pollen wall pattern (Figure 1).

\section{The Viability of abcg9 abcg31 Pollen Is Significantly Reduced under Normal Growth Conditions and Is Recovered by ABCG9 Expression}

The shriveled shape of abcg9-1 abcg31-1 pollen grains led us to suspect that the mutant pollen does not function normally. To test 
this possibility, we performed a pollen viability assay by staining the pollen with fluorescein diacetate (FDA) and propidium iodide (PPI; Regan and Moffatt, 1990). About half of the pollen of the abcg9-1 abcg31-1 plants was dead, whereas the viability of abcg9-1 and abcg31-1 single mutant pollen did not differ from that of the wild type (Figure 2A; Supplemental Figure 5). To confirm that the striking pollen phenotype of the double knockout was caused by mutation of ABCG9 and ABCG31 and not by any other reason, we also generated abcg9-1 abcg31-2 by crossing abcg9-1 with abcg31-2. The homozygous knockout plants were identified using genomic DNA-PCR (Supplemental Figure 4A). We found a similar level of reduction in the pollen viability of abcg9-1 abcg31-2 to that of abcg9-1 abcg31-1 (Supplemental Figure 5). As both abcg9-1 abcg31-1 and abcg9-1 abcg31-2 mutants exhibited a similar phenotype, we used abcg9-1 abcg31-1 mutant plants for further analysis.

The reduced pollen viability of the abcg9 abcg31 plants was

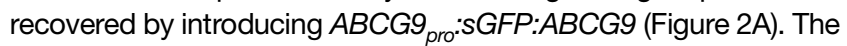
pollen viability of lines 1 (Compl-1) and 3 (Compl-3) was completely recovered to wild-type levels (no significant difference), whereas that of line 2 (Compl-2) was partially recovered. The recovery levels of the phenotype in complementation lines matched well with the fluorescence intensity from sGFP:ABCG9 in the anthers (Figure 2B); lines with high levels of fluorescence intensity in the anthers exhibited high recovery rates in pollen viability.

\section{The Phenotype of abcg9-1 abcg31-1 Pollen Varies Depending on the Environmental Conditions}

To examine whether the manifestation of the abcg9 abcg31 pollen phenotype depends on environmental conditions, two different phenotypic tests were conducted. First, pollen viability was tested again under humidity conditions $(70 \%)$ much higher than those in our greenhouse (20 to $30 \%$ ). The plants were grown in a chamber of intermediate humidity (50\%) until flowering, and then half were transferred to 20 to $30 \%$ humidity conditions and the other half to $70 \%$ humidity conditions. Within 1 week, pollen viability was assayed for pollen collected from open flowers. While the pollen viability of abcg9-1 abcg31-1 was only 50\% under 20 to $30 \%$ humidity, it recovered to $\sim 80 \%$ under $70 \%$ humidity. The pollen viability of the wild type was higher than $90 \%$ in both conditions (Figure 3A).

Cold shock is another environmental stress that affects pollen viability and, consequently, seed yield (Lee and Lee, 2003). We investigated the viability of wild-type and abcg9 pollen after cold treatment. The viability of abcg9-1 or abcg9-2 single knockout pollen was reduced to $\sim 70 \%$ by cold treatment, whereas that of wild-type pollen was barely affected (Supplemental Figure 6).

The decrease in mutant pollen viability under cold stress suggests that cold shock might also reduce the seed yield of the mutants. To test this possibility, wild-type, single knockout, and double knockout plants were grown in the greenhouse $\left(22^{\circ} \mathrm{C}\right)$ for 4 weeks, and half of each genotype was incubated at $4.5^{\circ} \mathrm{C}$ for $7 \mathrm{~d}$ and returned to the greenhouse. The siliques from the cold shock-treated and nontreated plants were harvested after 3 weeks. Under normal conditions, all genotypes of plants tested produced as many as 40 seeds per silique (Figure 3B, Normal), indicating that fertilization was normal in the mutant plants. This is most likely due to the large excess of pollen grains compared with the number of ovules; thus, all of the mutant ovules were fertilized even when about half of the pollen grains were dead (Figure 2A). Cold treatment decreased the seed yield of all plants. However, the seed yield of abcg9-1 abcg31-1 plants was reduced to the greatest extent and corresponded to only 20 to $30 \%$ of that obtained from wild-type and single knockout plants (Figure 3B, Cold).
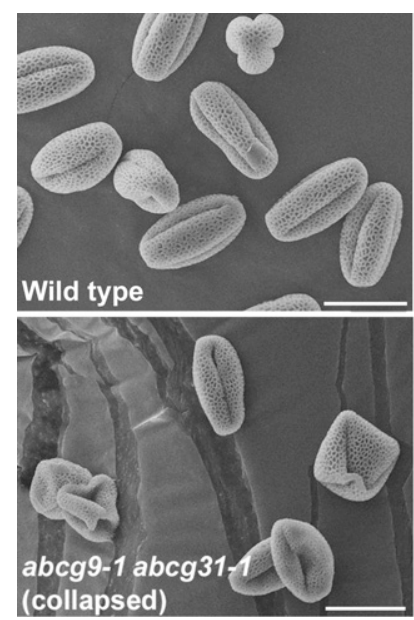
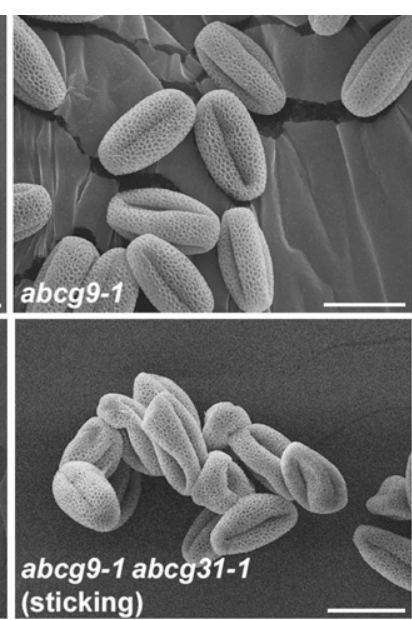

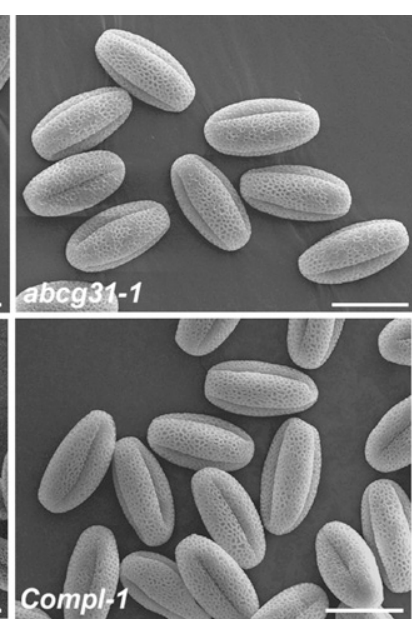

Figure 1. Air-Exposed abcg9-1 abcg31-1 Pollen Grains Exhibit a Shriveled Shape and a Tendency to Clump Together.

Scanning electron microscopy images show nonfixed mature pollen grains from 5- to 6-week-old wild-type, abcg9-1, abcg31-1, and abcg9-1 abcg31-1 plants and from abcg9-1 abcg31-1 plants complemented with ABCG9 driven by the ABCG9 promoter (Compl-1). In contrast with pollen from the wild type or single knockout mutants (abcg9-1 and abcg31-1), that from abcg9 abcg31 double knockout plants (abcg9-1 abcg31-1) was shriveled and collapsed or clumped together (sticking). This aberrant phenotype was recovered in the complemented line (Compl-1). Bars $=20 \mu \mathrm{m}$. 
A
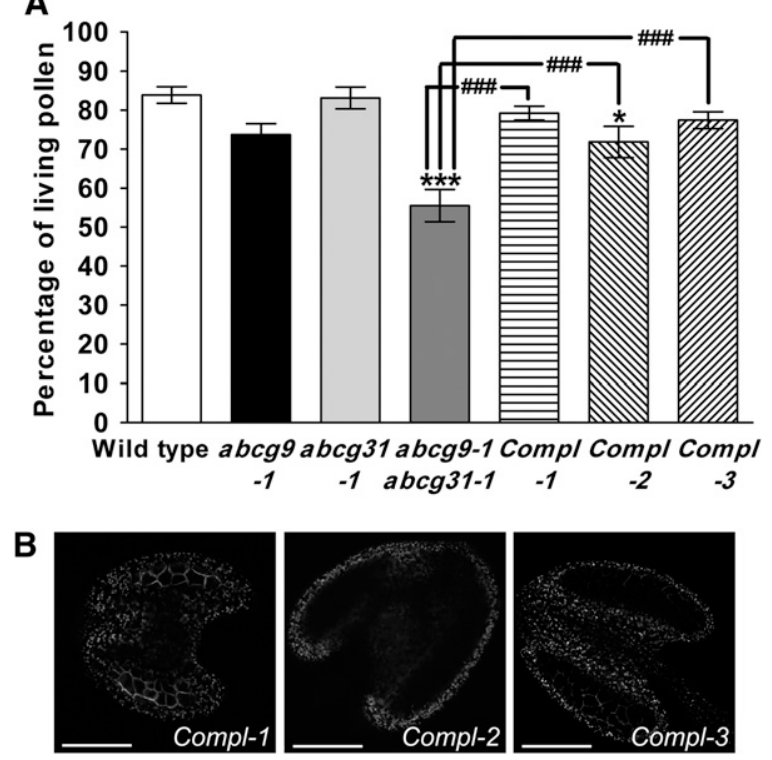

Figure 2. Recovery of Pollen Viability in abcg9-1 abcg31-1 Plants Complemented with ABCG9 Expression Driven by the ABCG9 Promoter.

(A) Pollen viability. Pollen from 4- to 5-week-old wild-type, abcg9-1, abcg31-1, and abcg9-1 abcg31-1 plants, and from abcg9-1 abcg31-1 plants expressing

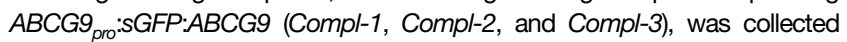
and stained using FDA and PPI, and the number of living (FDA-stained) and dead (PPI-stained) pollen grains was determined. Pollen viability is presented as the percentage of living pollen. The results from 30 flowers were combined (two flowers per count). Data represent means \pm SE of three biological replicates. Statistical comparison was performed using one-way ANOVA followed by Dunnett's multiple comparison test: ${ }^{\star} \mathrm{P}<0.05$ and ${ }^{\star \star \star} \mathrm{P}<0.001$ (compared with the wild type), and \#\#\# < 0.001 (compared with abcg9-1 abcg31-1). (B) Representative images for GFP:ABCG9 of anthers from Compl-1, Compl-2, and Compl-3. Bars $=100 \mu \mathrm{m}$.

\section{$A B C G 9$ and $A B C G 31$ Are Highly Coexpressed in Anthers}

The specific expression of $A B C G 9$ and $A B C G 31$ in stamens suggested by the in silico database was confirmed by quantitative RT-PCR (qRT-PCR) analysis, promoter- $\beta$-glucuronidase (GUS) assays, and GFP-tagged protein localization (Figure 4). ABCG9 transcripts were detected by qRT-PCR mainly in the flowers and siliques but were barely detectable in vegetative tissues (Figure 4A). ABCG9 transcript levels were high between stages 9 and 12 of flower development and then declined during the later stages. This finding is in good agreement with data from the in silico microarray database showing that $A B C G 9$ expression peaks at stages 10 and 11 and then decreases later (Supplemental Figure 1). To confirm this result, a promoter-GUS construct was produced by fusing the 2-kb fragment directly upstream of the $A B C G 9$ start codon to the GUS reporter gene. In the T1 transgenic plants, a GUS signal was found in the anthers and developing siliques (Figures 4B and 4C; Supplemental Figure 7A). When the siliques were cleared, the chalazal region of the developing seeds was also found to exhibit a GUS signal (Supplemental Figure 7C), which matches the in silico data (Supplemental Figure 1). In young seedlings of T2 plants, the GUS signal was not detected (Supplemental Figure 7E). Next,
ABCG9 localization in anther tissue was examined in the abcg9

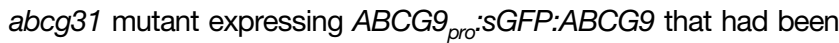
used for the complementation assay (Figures 1 and 2). Interestingly, sGFP signal occurred mostly in the tapetal cell layer (Figure 4E). The $A B C G 9_{\text {pro }}$ :SGFP:ABCG9 signal appeared not only in a spatially specific manner but also in a temporally specific manner. The signal
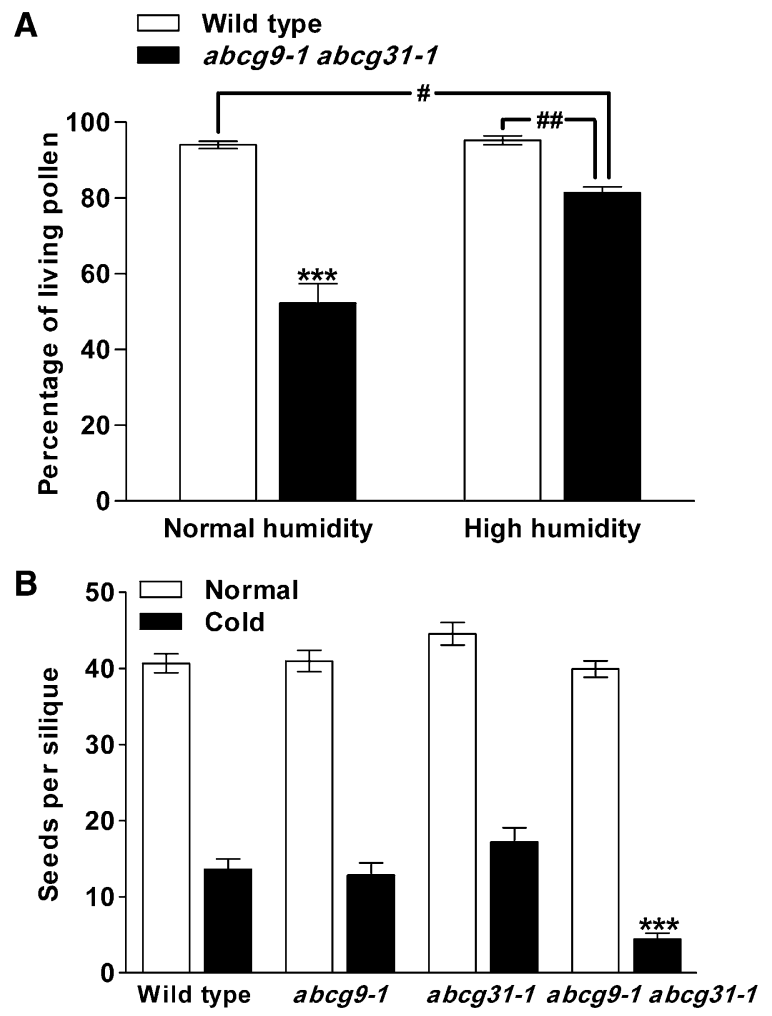

Figure 3. Phenotypes of abcg9-1 abcg31-1 Plants under Different Environmental Conditions.

(A) Pollen viability under normal or high humidity. Pollen grains were collected from 4-week-old plants incubated under normal (20 to $30 \%)$ or high $(70 \%)$ humidity, and pollen viability was examined as described in Figure 2. The results from eight (normal humidity) or nine (high humidity) flowers were combined. Data represent means \pm SE of two biological replicates. Statistical comparison was performed using one-way ANOVA followed by Tukey's multiple comparison test: ${ }^{* \star *} \mathrm{P}<0.001$ (compared with the wild type under normal or high humidity and abcg9-1 abcg31-1 under high humidity), \#P $<0.05$ and \#\# $<0.01$ (compared with abcg9-1 abcg31-1 under high humidity).

(B) Seed yield under normal growth conditions or after cold shock. Fourweek-old plants (i.e., in the flowering stage) were incubated at $4.5^{\circ} \mathrm{C}$ for $7 \mathrm{~d}$. Three weeks later, four siliques (normal) and three siliques (cold) were harvested from each plant, and their seed numbers were counted. The results from 31 (wild-type), 28 (abcg9-1), 25 (abcg31-1), and 53 (abcg9-1 abcg31-1) plants for the normal condition and 33 (wild-type), 30 (abcg9-1), 27 (abcg31-1), and 58 (abcg9-1 abcg31-1) plants for the cold shock treatment were combined. Data represent means \pm SE of three biological replicates. Statistical comparison was performed using one-way ANOVA followed by Tukey's multiple comparison test: ${ }^{* \star *} \mathrm{P}<0.001$ (compared with all genotypes under normal conditions and the wild type and single knockouts after cold shock). 

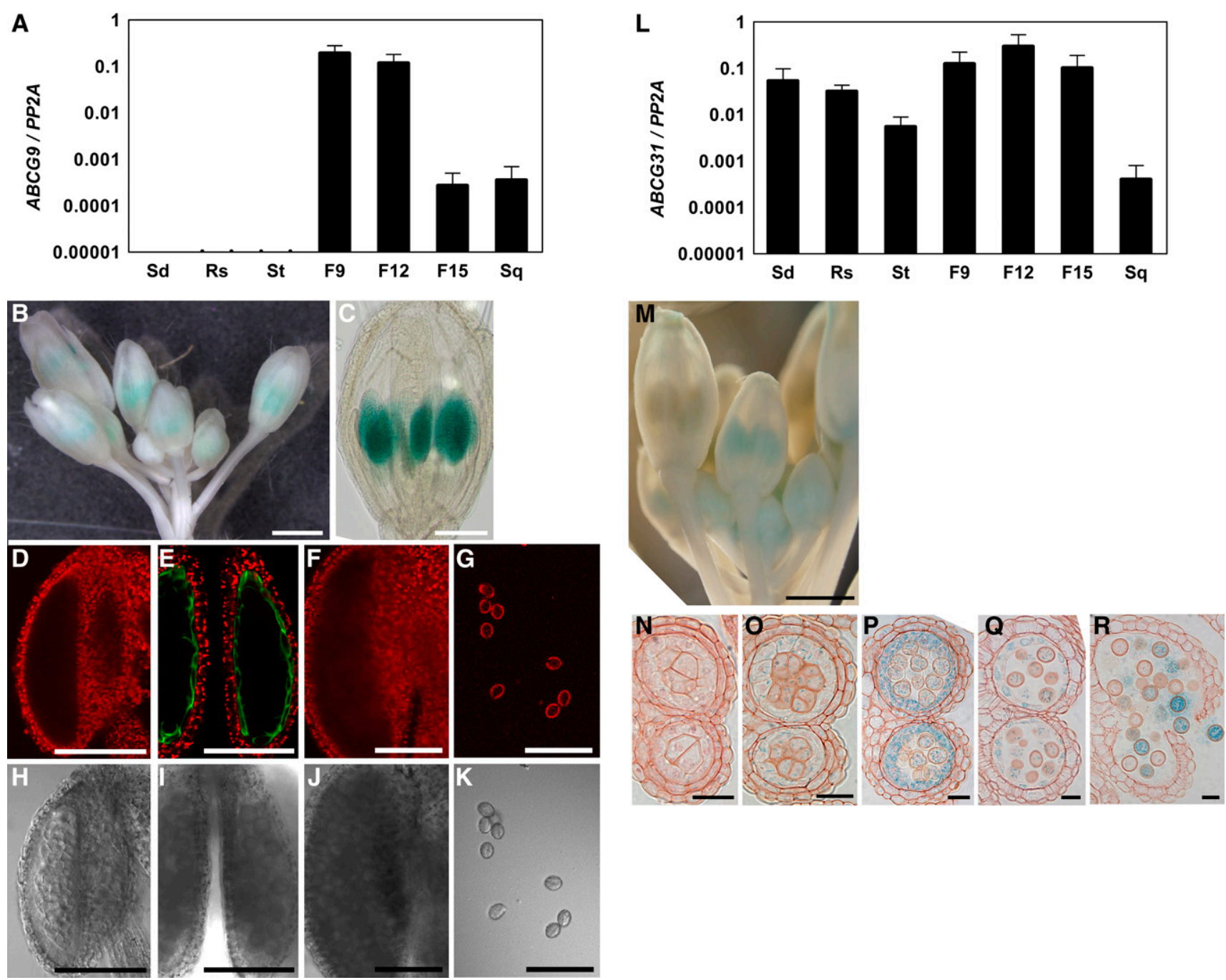

Figure 4. Tissue-Specific Expression of $A B C G 9$ and $A B C G 31$.

(A) qRT-PCR analysis of ABCG9 transcripts in 2-week-old wild-type seedlings (Sd) and various tissues in 4-week-old wild-type plants, including rosettes (Rs), stems (St), flowers at developmental stages 1 to 9 (F9), flowers at developmental stages 10 to 12 (F12), flowers at developmental stages 13 to 15 (F15), and green siliques (Sq). ABCG9 transcript levels were normalized against those of PROTEIN PHOSPHATASE 2A SUBUNIT A3 (PP2A). Data represent means \pm SE of two biological replicates.

(B) GUS expression in the anthers of a flower transformed with $A B C G 9_{\text {pro }}: G U S$. Bar $=1 \mathrm{~mm}$.

(C) Anther-specific staining of GUS in a flower transformed with $A B C G 9_{\text {pro: }}$ GUS. GUS-stained flowers were cleared using chloral hydrate (chloral hydrate:water:glycerol, 8:2:1, w/v/v). Bar $=200 \mu \mathrm{m}$.

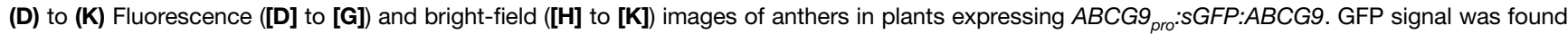
exclusively in the tapetum of the stage 10 anther ([E] and [I]). No GFP signal was present in the anther at the tetrad stage ([D] and [H]), after tapetum degeneration ([F] and $[\mathbf{J}]$ ), or in mature pollen grains ([G] and $[\mathbf{K}])$. Chlorophyll autofluorescence is shown for visualization of anther tissue in (D) to (G). Bars $=100 \mu \mathrm{m}$.

(L) qRT-PCR analysis of $A B C G 31$ transcripts in various tissues of wild-type plants. The cDNA and the normalizing control used are the same as those used for $A B C G 9$ analysis (A). Data represent means \pm SE of two biological replicates.

(M) GUS expression in the anthers of a flower bud transformed with $A B C G 31_{\text {pro: }}$ GUS. Bar $=0.5 \mathrm{~mm}$.

(N) to (R) Semithin sections of anthers from ABCG31 $1_{\text {pro }}$ GUS plants. GUS expression was not detected at the pollen mother cell stage (N) but appeared in both the tapetum and pollen of the tetrad stage $(\mathbf{O})$, was highest at the unicellular stage $(\mathbf{P})$, and then decreased at the tapetal degeneration stage $(\mathbf{Q})$, finally occurring in only some pollen at the mature stage (R). Bars $=20 \mu \mathrm{m}$. 
was apparent from the microspore stage until tapetum degeneration (Figures $4 \mathrm{E}$ and $4 \mathrm{I}$ ) but not during earlier or later stages (Figures $4 \mathrm{D}$ and $4 \mathrm{H}, 4 \mathrm{~F}$ and $4 \mathrm{~J}$, and $4 \mathrm{G}$ and $4 \mathrm{~K}$, respectively).

$A B C G 31$ transcripts were detected in all tissues but were most abundant in flowers at stages 10 to 12 (F12), when ABCG9 expression was also high (Figures $4 \mathrm{~A}$ and $4 \mathrm{~L}$ ). To observe the expression pattern of $A B C G 31$ in flowers, $A B C G 31$ promoterGUS assays were performed. The fragment comprising the region from $2 \mathrm{~kb}$ upstream of the ABCG31 start codon to the second exon was fused to GUS. In the flowers of T1 transgenic plants, the GUS signal was found mainly in the anthers (Figure $4 \mathrm{M})$. The GUS signal from developing siliques (Supplemental Figure 7B) was in the maternal tissues of siliques but not in the seed itself (Supplemental Figures 7D). Semithin sections of anthers revealed that $A B C G 31$ is expressed in the pollen and tapetum of anthers from the tetrad stage to the tapetal degeneration stage (Figures $4 \mathrm{O}$ to $4 \mathrm{Q}$ ) and in the pollen at the mature stage (Figure 4R). The GUS signal was most prominent at the unicellular stage and was much higher in the tapetum than in the pollen (Figure 4P). In young seedlings, the patterns of GUS expression were variable, even between individual plants of a single line; about two-thirds of the lines examined displayed the signal mainly in the whole shoots, including the vasculature, and most lines exhibited signal in the root vasculature, even though the expression levels varied (Supplemental Figure 7F). When the signal was weaker, the expression was more restricted to the tips of cotyledons and the basal parts of leaf petioles (Supplemental Figure 7G). ABCG31 was previously reported to be involved in abscisic acid (ABA) signaling (Francia et al., 2008). Such variation in GUS pattern in the ABCG31pro: GUS lines is possibly due to differences in the ABA response. Together, these expression assays for ABCG9 and ABCG31 revealed that the tissue-specific expression pattern of $A B C G 9$ matched that of $A B C G 31$ in the developing anther and was consistent with information from the in silico coexpression database (Supplemental Data Set 2), supporting the possibility that ABCG9 and ABCG31 participate in a similar process.

\section{ABCG9 and ABCG31 Localize to the Plasma Membrane}

ABCG9 and ABCG31 have no putative targeting sequences known to direct the proteins to any intracellular compartment and are predicted to localize to the plasma membrane. To determine the precise intracellular localization of ABCG9 and ABCG31, we expressed sGFP-tagged ABCG9 or sGFP-tagged ABCG31 driven by the cauliflower mosaic virus $35 \mathrm{~S}$ promoter in Nicotiana benthamiana cells using an agroinfiltration method (Figures $5 \mathrm{~A}$ to $5 \mathrm{C}$ and $5 \mathrm{D}$ to $5 \mathrm{~F}$, respectively). The protoplasts isolated from leaves transformed with either sGFP-ABCG9 or sGFP-ABCG31 exhibited green fluorescence at the plasma membrane. Next, ABCG9 localization in anther tissue was examined in the $A B C G 9_{\text {pro:sGF: }}$ ABCG9-expressing abcg9 abcg31 Arabidopsis plants used for the complementation assay (Figures 1 and 2). Interestingly, sGFP signal was found exclusively in the tapetal cell layer (Figure 4E) and was localized to the plasma membrane (Figures $5 \mathrm{G}$ and $5 \mathrm{H}$ ), which is consistent with the plasma membrane localization found in cells transformed with constructs driven by the cauliflower mosaic virus $35 \mathrm{~S}$ promoter (Figures 5A and 5B).

\section{The Pollen Coat of abcg9-1 abcg31-1 Plants Is Incomplete}

Scanning electron microscopy images of abcg9-1 abcg31-1 pollen revealed no alteration in exine patterns (Figure 1), suggesting that the pollen walls are normal and are not the cause of the pollen shriveling or decrease in viability. As we did not observe any defects in pollen development (Supplemental Figure 8), we suspected that the pollen coat was defective and thus might have caused the defects in pollen. We tested this possibility using transmission electron microscopy (TEM). Just before anthesis, at the time when flowers bear mature pollen grains, the overall external appearance of abcg9-1 abcg31-1 pollen did not differ from that of the wild type (Figures 6A and 6C). However, differences were apparent in the pollen coat. In the wild type, the pollen coat of mature pollen grains was fully filled between neighboring baculae, which are column-like structures of sexine (outer exine; Figure 6B). In abcg9-1 abcg31-1 plants, however, the pollen coat was not completely filled and often was interspersed with vesicular or linear electron-lucent structures (Figure 6D, arrowheads). This irregular coat structure of the double knockout pollen resembled that of the immature pollen of the wild type at the beginning stage of pollen coat accumulation (Figure 6E; Supplemental Figure 9), which also contained similar electronlucent structures (Figure 6F, arrowheads).

\section{The Steryl Glycoside Content in the Pollen of abcg9-1 abcg31-1 Plants Is Reduced}

The unusual structures we found in the abcg9-1 abcg31-1 pollen coat may reflect reduced pollen coat contents or altered pollen coat composition in the mutant. As the pollen coat mainly consists of sterols, especially steryl ester, we performed sterol analysis using whole pollen grains. Although we expected that the mutant would have a reduced steryl ester content, the difference in free sterol and steryl ester contents between wild-type and abcg9-1 abcg31-1 pollen was not significant (Figure 7, inset graph; Supplemental Figure 10). By contrast, we found that abcg9-1 abcg31-1 pollen contained only about half of the amount of steryl glycosides present in wild-type or single knockout mutant pollen (Figure 7; see Supplemental Figure 11 for additional information on MW470 compounds). Another component enriched in the pollen coat is wax (Fiebig et al., 2000). However, the wax contents of wild-type and double knockout mutant pollen did not differ much, at 20.1 and $20.2 \mu \mathrm{g} / \mathrm{mg}$ dry weight, respectively (Supplemental Figure 12). Furthermore, the mutant pollen did not differ from wild-type pollen in the level of brassinosteroids, steroid hormones that are greatly enriched in pollen (Grove et al., 1979), with values of 219 and $199 \mathrm{pg} / \mathrm{mg}$ dry weight for wild-type and abcg9-1 abcg31-1 pollen, respectively (Supplemental Table 1).

\section{A Steryl Glycoside-Deficient Mutant Exhibits Reduced Pollen Viability and Incomplete Pollen Coats, Similar to abcg9 abcg31}

In Arabidopsis, the biosynthesis of steryl glycosides is catalyzed mainly by two membrane-bound UDP-glucose:sterol glycosyltransferases, UGT80A2 and UGT80B1. Recently, DeBolt et al. (2009) generated a double knockout mutant, ugt80A2 ugt80B1, and showed that the mutant had significantly less steryl glycoside 

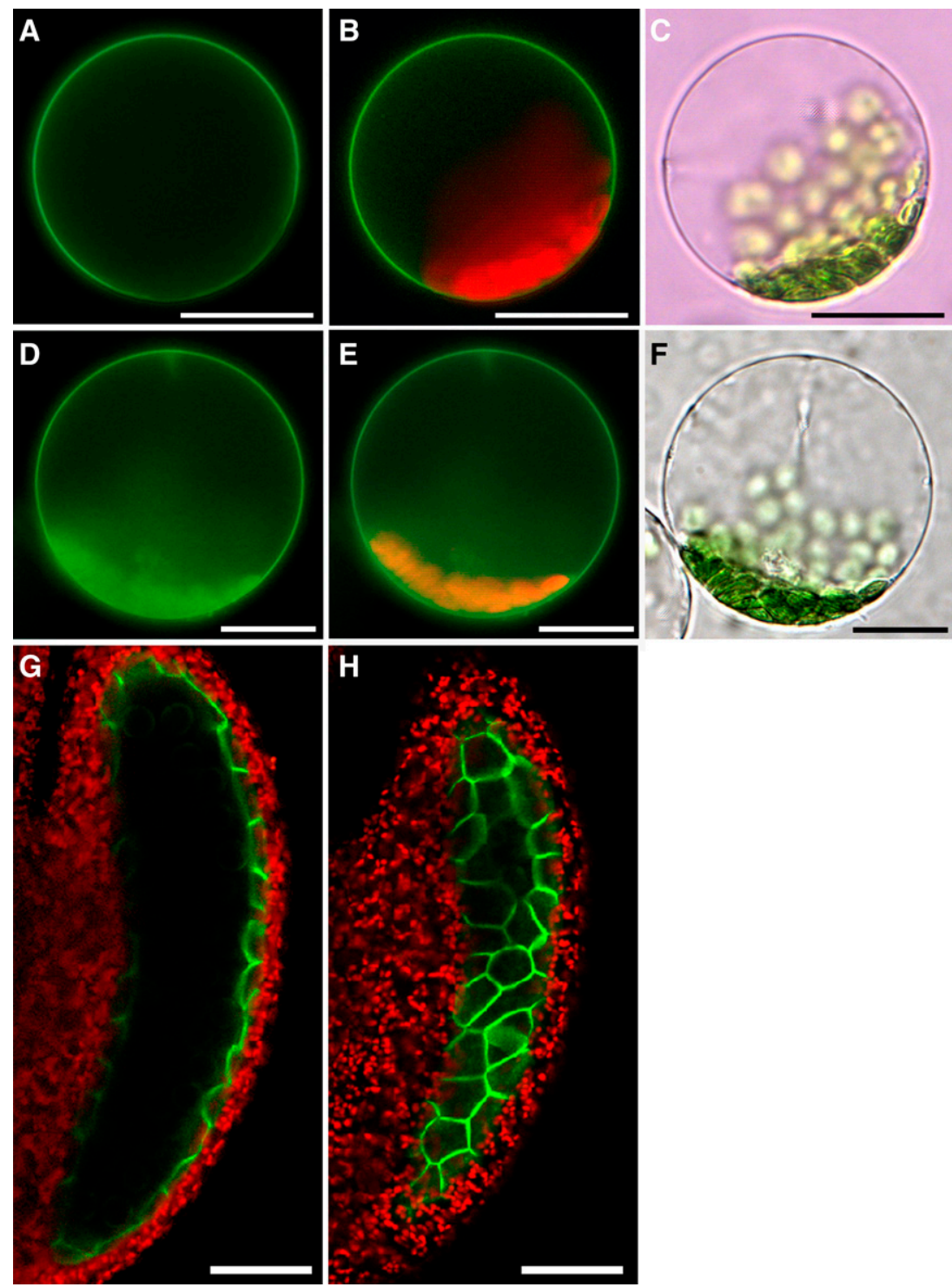

Figure 5. Plasma Membrane Localization of ABCG9 and ABCG31.

(A) to (F) ABCG9 and ABCG31 localization in N. benthamiana leaf cells. Protoplasts were isolated from N. benthamiana leaves transformed with $35 S_{\text {pro: }}$

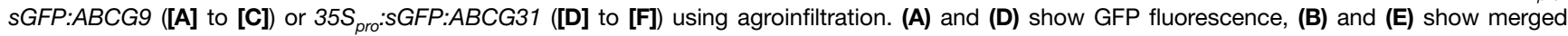
images of GFP and chlorophyll autofluorescence, and (C) and (F) show bright-field images. Bars $=20 \mu \mathrm{m}$.

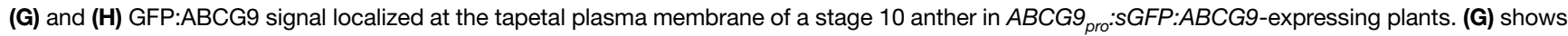
an image taken from a focal plane at the center of the anther, and $\mathbf{( H )}$ shows an image taken from a focal plane at a distal region of the same anther shown in (G). Chlorophyll autofluorescence is shown for visualization of anther tissue. Bars $=50 \mu \mathrm{m}$.

in the leaves, stems, inflorescences, and siliques. If the reduced steryl glycoside content in abcg 9 abcg 31 mutant pollen was the underlying cause for the reduction in pollen viability, the ugt80A2 ugt80B1 mutant, which is deficient in steryl glycoside biosynthesis, should also display a similar phenotype. To test this possibility, we performed a pollen viability assay under normal growth conditions and found that, like the abcg9 abcg31 mutants (Figure 2), the ugt80A2 ugt80B1 mutant pollen exhibited reduced viability (Figure 8A). Moreover, we found that the pollen coat of the ugt80A2 ugt80B1 mutant was often incomplete, containing vesicular or linear electron-lucent structures more frequently than that of the wild type (Figures $8 \mathrm{~B}$ and $8 \mathrm{C}$ ), although the defects were not as prominent as in pollen of the abcg9 abcg31 mutant (Figures 6B and 6D).

\section{DISCUSSION}

Here, we studied two ABC transporters that are highly expressed in Arabidopsis tapetal cells (Figures 4E and 4P) and found that they are important for normal pollen coat development and, consequently, for pollen fitness. The deletion of the two $A B C$ 

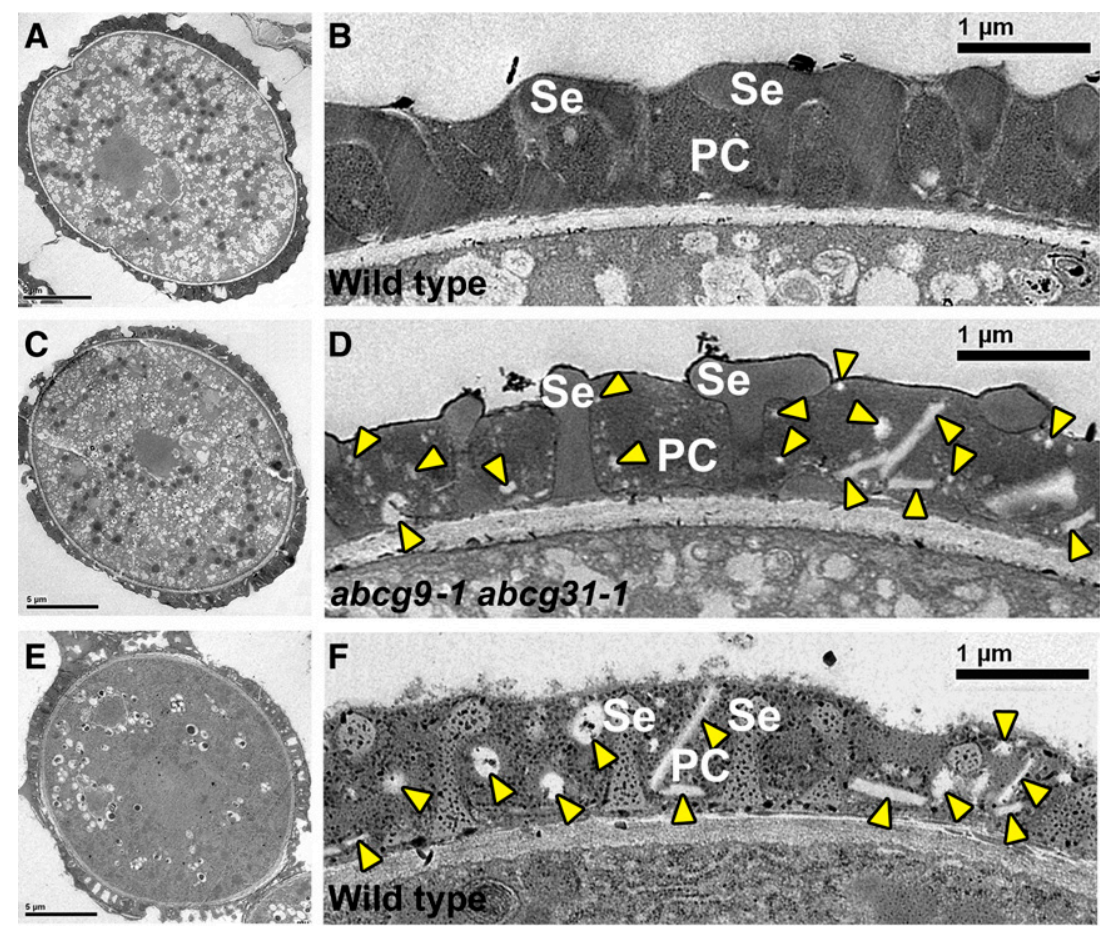

Figure 6. Incomplete Pollen Coat Formation of abcg9-1 abcg31-1 Plants.

Pollen grains were observed using TEM: wild-type pollen (Columbia-0 ecotype; [A], [B], [E], and [F]) and abcg9-1 abcg31-1 pollen (Columbia-0 ecotype background; [C] and [D]).

(A) and (C) The overall appearance of wild-type and abcg9-1 abcg31-1 pollen grains was similar immediately before anthesis. Bars $=5 \mu \mathrm{m}$.

(B) and (D) TEM images of wild-type and abcg9-1 abcg31-1 pollen coats before anthesis. Bars $=1 \mu \mathrm{m}$.

(B) Wild-type pollen with a normal pollen coat (PC), which fills the cavity between neighboring baculae of the sexine (Se).

(D) abcg9-1 abcg31-1 pollen, which is not completely filled with pollen coat and contains many irregular structures between the baculae. Arrowheads indicate abnormal vesicular and linear, electron-lucent structures in the coat of abcg9-1 abcg31-1 pollen.

(E) Overall structure of a wild-type pollen grain at the initial coat deposition stage. Pollen coat material is beginning to cover the surface of pollen grains. Bar $=5 \mu \mathrm{m}$.

(F) The pollen coat of the wild-type plant at the initial coat deposition stage contains vesicular and linear structures (arrowheads) between the baculae of the sexine, similar to the pollen coat shown in (D). Bar $=1 \mu \mathrm{m}$.

transporters, $A B C G 9$ and $A B C G 31$, resulted in pollen that failed to form complete pollen coats (Figure 6) and reduced the ability of pollen to withstand environmental stresses (Figures 1 to 3). The morphological defect in the pollen coat was accompanied by a reduction in steryl glycoside content in the abcg9 abcg31 mutant pollen (Figure 7), and these defects were phenocopied by ugt80A2 ugt80B1, a steryl glycoside-deficient mutant (Figure 8). These results indicate that the two Arabidopsis ABC transporters contribute to pollen coat assembly. Since the transporters are localized to the plasma membrane (Figure 5) and belong to the subfamily of transporters known to transport lipidic molecules in many organisms, they most likely transport steryl glycosides or unidentified related compounds from the tapetal plasma membrane to the surface of pollen. These results suggest that pollen coat deposition may not be a simple one-step event that occurs at the time of tapetum degeneration but requires steps that are mediated by transporters that require energy and that, without these steps, pollen viability is impaired.

\section{Reduced Pollen Viability in abcg9 abcg31 Plants Is Related to a Defective Pollen Coat}

Exposure to dry air, which results in water loss, caused abcg9 abcg31 mutant pollen grains to shrivel up and collapse (Figure 1), leaving half of them dead (Figure 2; Supplemental Figure 5). However, pollen viability of the mutant was almost recovered to wildtype levels after exposure to high humidity (Figure 3A), suggesting that the reduced pollen viability was mainly due to excessive water loss through the pollen surface.

Pollen viability is often affected by a defect of the pollen wall or coat. In the case of abcg9 abcg31 mutant pollen, the cause of the increased water loss was a defect in the pollen coat and not in the pollen wall. When observed by scanning electron microscopy or TEM, the pollen wall structure of the abcg 9 abcg31 mutant was not different from that of the wild type (Figures 1 and 6 , respectively). However, the pollen coat of the mutant was abnormal, containing many electron-lucent vesicular or linear structures (Figure 6D, arrowheads). Similar structures could also 


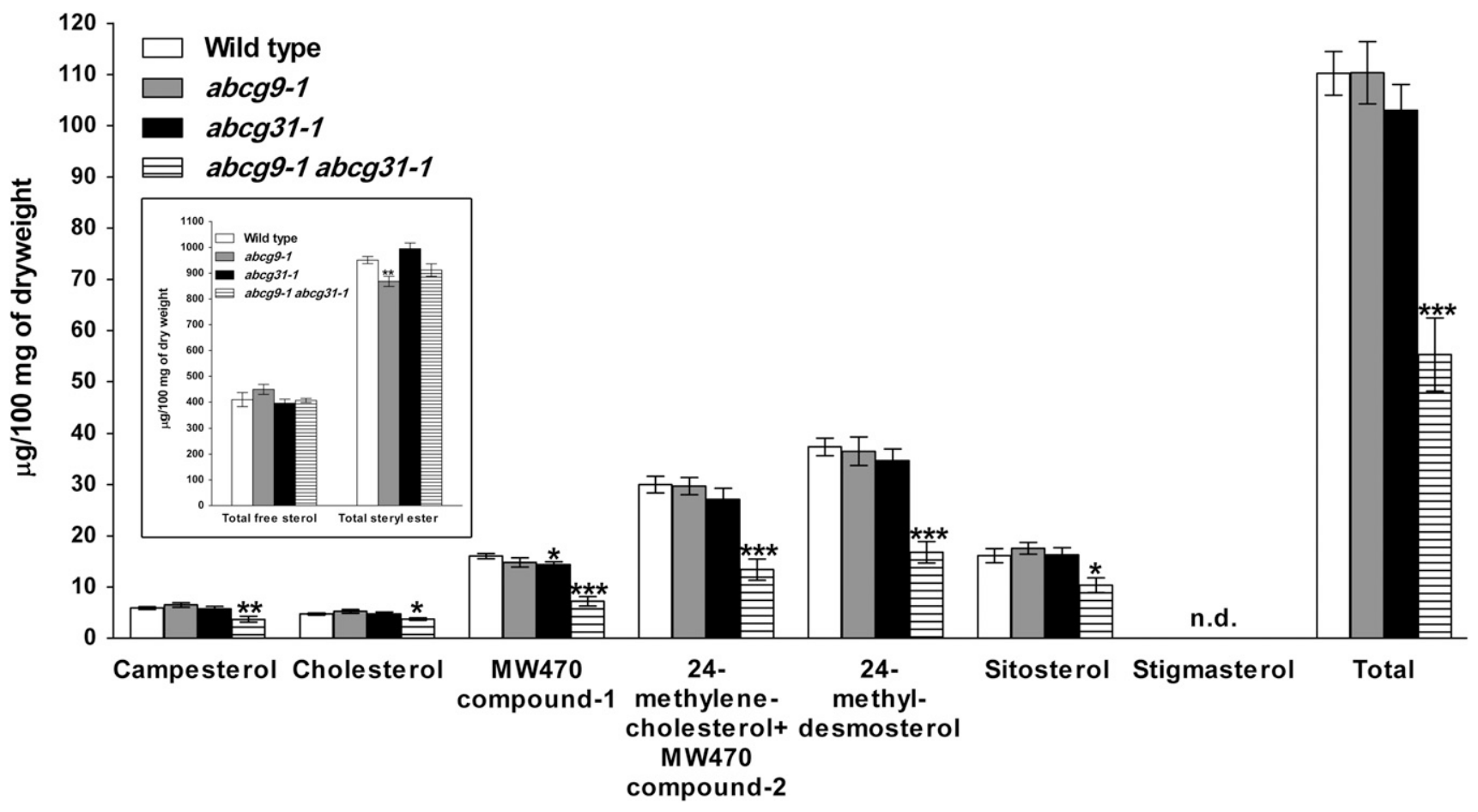

Figure 7. Reduced Steryl Glycoside Content in the Pollen of abcg9-1 abcg31-1 Plants.

Pollen was collected from 4- to 5-week-old wild-type, abcg9-1, abcg31-1, and abcg9-1 abcg31-1 plants. The steryl glycoside fraction was extracted and separated according to sterol moiety using GC-MS. From $\sim 50 \mathrm{mg}$ of pollen sample, three independent lipid extractions and separations were executed. MW470 compounds 1 and 2 are unidentified molecules. Possible candidates are described with their mass spectra in Supplemental Figure 11. The inset graph shows total free sterol and steryl ester contents in the corresponding genotypes. Data represent means \pm SE of two biological replicates (Student's $t$ test: ${ }^{*} \mathrm{P}<0.05$, ${ }^{* *} \mathrm{P}<0.01$, and ${ }^{\star * *} \mathrm{P}<0.001$, compared with the wild type). n.d., not detected.

be observed in the wild type at the beginning of pollen coat deposition (Figure 6F, arrowheads), but they were replaced by a more compact and highly ordered structure as the pollen matured and dehydrated as described previously (Piffanelli et al., 1998). Therefore, the increased sensitivity of mutant pollen to stress may be a result of impaired pollen coat maturation.

\section{ABCG9 and ABCG31 Are Important for the Transfer of Steryl Glycosides from the Tapetum to the Pollen Surface}

Based on three major findings, we suggest that ABCG9 and ABCG31 play an important role in the transfer of steryl glycosides from the tapetum to the pollen surface. The first finding involves the spatial and temporal pattern of expression of these genes. The genes are predominantly expressed at the tapetum rather than in the pollen (Figures 4 and 5), but their absence causes a defect in the pollen coat, which implies that they are involved in the transfer of materials from the tapetum to the pollen. Temporally, the expression of these genes peaks prior to tapetal degeneration, immediately before the completion of pollen coat formation, at a time when pollen coat materials might be transferred. The second finding involves our analysis of lipidic molecules of pollen. The abcg9 abcg31 mutant pollen had only half as much steryl glycosides as the wild-type pollen but normal levels of other pollen coat materials (i.e., free sterols, steryl esters, and wax) and brassinosteroids. Third, many members of the ABCG subfamily in various organisms have been shown to participate in the transport of sterols and related compounds. Human ABCG2, originally reported to be an effective anticancer drug transporter, can also transport cholesterol and steroid hormones (Janvilisri et al., 2003). Mouse ABCG5/ABCG8 heterodimer transports cholesterol and plant sterols (Wang et al., 2008a). Mouse ABCG1 and ABCG4 promote cholesterol efflux (Wang et al., 2008b). Yeast full-size ABCG proteins AUS1 and PDR11 are required for sterol uptake (Wilcox et al., 2002). Other ABC subfamily members have also been suggested to transport steroidal glycosides; human ABCB1 and Magnaporthe oryzae (rice blast fungus) Abc1 are responsible for the export of the steroidal glycosides digoxin or digitoxin from the cell, thus protecting it from chemical toxicity (Tanigawara et al., 1992; Patkar et al., 2012). The multiple reports of $A B C$ proteins functioning in the transfer of sterol-related compounds support our conclusion that ABCG9 and ABCG31 participate in the transport of steryl glycosides either directly or indirectly. Such deposition of steryl glycosides on the pollen surface might facilitate the normal deposition of the remaining coat materials at the time of tapetal degeneration.

Among the $131 \mathrm{ABC}$ transporter proteins in Arabidopsis, the ABCG subfamily is the largest, containing 43 members (Verrier et al., 2008). Some of the ABCG proteins have been found to be important for the delivery of lipids to the cell surface. ABCG11 participates in 

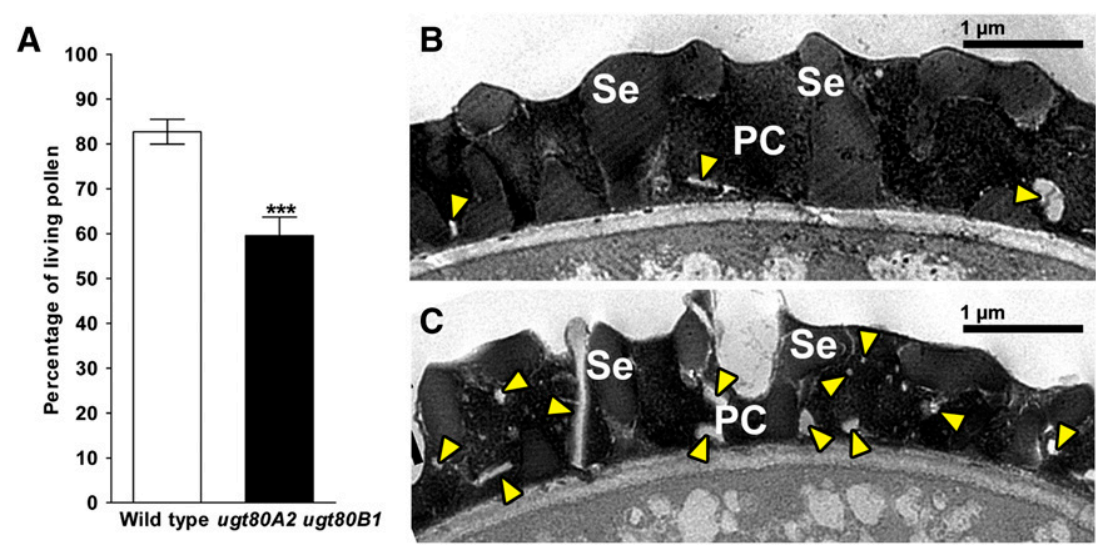

Figure 8. Reduced Pollen Viability and Incomplete Pollen Coat Formation of ugt80A2 ugt80B1 Plants.

(A) Pollen viability of wild-type (Wassilewskija ecotype) and ugt80A2 ugt80B1 (Wassilewskija ecotype background) plants. Pollen from 4-week-old plants was collected, and pollen viability was examined as described in Figure 2. The results from nine flowers from different plants were combined. Data represent means \pm SE of two biological replicates (Student's $t$ test: ${ }^{* \star *} P<0.001$ ).

(B) and (C) TEM images of wild-type and ugt80A2 ugt80B1 pollen coats before anthesis. The coat of ugt80A2 ugt80B1 pollen (C) contains many irregular structures, which are seldom seen in the coat of wild-type pollen (B). Arrowheads indicate abnormal vesicular and linear, electron-lucent structures in the coat of wild-type and ugt80A2 ugt80B1 pollen. PC, pollen coat; Se, sexine. Bars $=1 \mu \mathrm{m}$.

the transport of long-chain fatty acids, which are precursors of cutin, wax, and suberin (Bird et al., 2007; Luo et al., 2007; Panikashvili et al., 2007; Ukitsu et al., 2007). ABCG12 is involved in the transport of very long chain fatty acids, such as wax precursors (Pighin et al., 2004). ABCG13 is required for the normal formation of petal surface cutin (Panikashvili et al., 2011). ABCG32 is necessary for the transfer of aliphatic cutin monomers for the formation of the cuticular layer of the cell wall of leaves, stems, and petals (Bessire et al., 2011). ABCG26 contributes to the deposition of pollen exine on the pollen surface (Quilichini et al., 2010; Xu et al., 2010; Choi et al., 2011; Dou et al., 2011). However, the functions of $A B C G$ s are not restricted to lipid transfer, as many members of this group of proteins are also involved in biotic and abiotic stress responses, most likely by transporting terpenoids and their derivatives, as reported for Arabidopsis and other plants (Kang et al., 2011; Kuromori et al., 2011; Xi et al., 2012; Bienert et al., 2012; Crouzet et al., 2013). Despite numerous reports of $A B C$ proteins having roles in lipid and terpenoid transport, so far, plant transporters have not been reported to be involved in the transport of sterols or their derivatives.

Another possible function of the two transporters is to redistribute steryl glycoside at the tapetal plasma membrane and thereby regulate programmed cell death of the tapetum. In Pichia pastoris, steryl glycosides are known to be involved in pexophagy, a form of programmed cell death (Oku et al., 2003). We speculate that the altered distribution of steryl glycosides at the tapetal plasma membrane caused by the absence of ABCG9 and ABCG31 may delay tapetum degeneration, postponing also the release and deposition of pollen coat material. Such a delay would prevent the mutant pollen grain from completing pollen coat maturation before anther dehiscence. However, we did not observe a difference in the timing of mutant tapetum degeneration; thus, this possibility is not strongly supported by our experimental data.
Recently, Le Hir et al. (2013) suggested a role for ABCG9/ ABCG11/ABCG14 in sterol/lipid homeostasis during vascular development. This suggestion was based on the observations that knockout plants of these three genes have altered levels of sterols and other lipids, with reduced levels of expression of enzymes related to sterol homeostasis; that they are expressed in the phloem; and that sterols are found in the vasculature. However, direct evidence for the transport of sterols/lipids by any of these transporters awaits further studies.

\section{Steryl Glycosides May Be Important for Pollen Coat Assembly}

The incomplete pollen coat structure and cold/dry air sensitivity of abccg9 abcg31 pollen, together with their reduced steryl glycoside content, suggest that steryl glycosides are an important component in pollen coat assembly and thus contribute to the protection of pollen. Consistent with this interpretation, the ugt80A2 ugt80B1 mutant, which is deficient in steryl glycoside synthesis, exhibited similarly reduced pollen viability and incomplete pollen coats (Figure 8). However, compared with the phenotype of abcg9 abcg31 plants, that of ugt80A2 ugt80B1 plants is less pronounced. The difference in pollen viability or pollen coats between the wild type and the ugt80 double mutant is not as great as that for the abcg9 abcg31 mutant. This raises the question of whether compounds other than steryl glycosides are transported by these ABCG proteins. However, the levels of free sterols, steryl esters, waxes, and brassinosteroids did not differ much between mutant and wild-type pollen (Supplemental Figures 10 and 12 and Supplemental Table 1).

Another explanation for the difference in the severity of the phenotype between abcg 9 abcg31 and ugt80A2 ugt80B1 plants could be the presence of an alternative metabolic pathway or a metabolite that could compensate for the defect in the biosynthetic mutants. Indeed, in the ugt80A2 ugt80B1 mutant, the 
free sterol plus steryl ester content was $26 \%$ higher than in the wild type (DeBolt et al., 2009), while in the abcg9 abcg31 mutant, an increase of only $10 \%$ was observed (Figure 7, inset graph). Free sterols and steryl esters or other related lipids, therefore, may partially substitute for the function of steryl glycosides in the ugt80A2 ugt80B1 mutant, making the mutant phenotype less pronounced than that of abcg9 abcg31. However, we cannot rule out the possibility that ABCG9 and ABCG31 transport other substrates in addition to the sterols, since many $A B C$ transporters are known to transport multiple substrates (Rea, 2007). ABCG31 was once suggested to function in the $A B A$ response in guard cells (Francia et al., 2008). Although ABCG31 did not appear to be expressed in the guard cells of flowers or anthers in our reporter line, ABA may still be considered as a candidate substrate of ABCG31, as ABA is important for drought resistance and the abcg 9 abcg 31 mutant exhibited an aberrant phenotype in dry air. Unfortunately, there is no information on the function of $A B A$ in pollen viability or pollen coat formation.

In plant cells, steryl glycosides are mainly found at the plasma membrane (Hartmann and Benveniste, 1987), where they might regulate membrane properties (Ullmann et al., 1987; Palta et al., 1993; Warnecke et al., 1999). However, no direct evidence for the function or localization of steryl glycosides has been provided for pollen, either at the plasma membrane or in the coat. Previously, it was suggested that the steryl glycosides might act as primers for cellulose synthesis (Peng et al., 2002). Mature pollen grains and developing microspores have cellulosic compounds in the intine (inner pollen wall) and primexine, respectively, which are synthesized by the pollen itself. However, we could not detect any defect in the intine structure of the abcg9 abcg31 mutant using TEM. It is unlikely that ABCG9 or $A B C G 31$ is involved in primexine formation, either, since primexine is formed at the tetrad stage, long before the expression of $A B C G 9$ and $A B C G 31$ peaks. Therefore, steryl glycosides do not seem to contribute to pollen fitness by facilitating cellulose biosynthesis. Instead, our data suggest that steryl glycosides are involved in pollen coat maturation as one of the pollen coat components; the steryl glycoside content of pollen $(\sim 110 \mu \mathrm{g} /$ $100 \mathrm{mg}$ dry weight; Figure 7) is much higher than that of the leaf, stem, and inflorescence/silique ( 25,30 , and $35 \mu \mathrm{g} / 100 \mathrm{mg}$ dry weight, respectively; DeBolt et al., 2009). When normalized by cell surface area, the steryl glycoside content of pollen is estimated to be 8.8 times higher than that in the leaf (Supplemental Methods). Thus, at least some steryl glycosides are localized in the normal pollen coat, and a reduction in steryl glycoside content might compromise coat structure. The role of steryl glycoside during pollen coat deposition is unclear. Since the glucose moiety of steryl glycoside makes the sterol amphiphilic, we speculate that it might function as a surfactant that facilitates the mixing of various chemicals that make up the pollen coat.

In summary, we show here that ABCG9 and ABCG31 are involved in the pollen coat maturation process, possibly by regulating the distribution of steryl glycosides on the pollen surface, and thereby contribute to pollen fitness. Further investigations are needed to decipher the mechanisms by which lipids are deposited on the pollen surface and to pinpoint the roles of $A B C$ transporters in this process.

\section{METHODS}

\section{Plants and Growth Conditions}

Arabidopsis thaliana plants were grown under a 16 -h photoperiod at $22^{\circ} \mathrm{C}$ in a greenhouse with 20 to $30 \%$ humidity. Ecotype Columbia-0 was used, except for ugt80A2 ugt80B1, which was in a Wassilewskija background (DeBolt et al., 2009).

\section{Isolation of T-DNA Knockout Mutants}

The abcg9 knockout mutants, abcg9-1 (SALK_045984) and abcg9-2 (SALK_047133), were obtained from the Salk Institute Genomic Analysis Laboratory. The abcg31 knockout mutants, abcg31-1 (WiscDsLox303G10) and abcg31-2 (GABI_064C11), were obtained from the Salk Institute Genomic Analysis Laboratory and GABI-Kat, respectively. The T-DNA insertion site of each mutant is presented in Supplemental Figures 2 and 3. The T-DNA insertion was verified by genomic DNA-PCR. RT-PCR analysis confirmed that abcg9 and abcg31 mutants did not produce ABCG9 and ABCG31 transcripts, respectively. The $\beta-T U B U L I N$ and UBIQUITIN genes were used as internal controls for $A B C G 9$ and $A B C G 31$, respectively.

The abcg 9 abcg31 double knockout mutants were generated from crosses between abcg9-1 and abcg31-1 (abcg9-1 abcg31-1) or between abcg9-1 and abcg31-2 (abcg9-1 abcg31-2). The T-DNA insertion in each gene was verified by genomic DNA-PCR, and the absence of both ABCG9 and ABCG31 transcripts in the double knockout mutant was confirmed by RT-PCR.

\section{Promoter-GUS Expression Assay}

For promoter-GUS expression, ABCG9pro:GUS and ABCG31pro:GUS constructs were generated. Construct information is available in Supplemental Table 3. Each final construct was introduced into wild-type Arabidopsis using the Agrobacterium tumefaciens-mediated floral dipping method (Clough and Bent, 1998).

To observe promoter-GUS expression in flowers and developing siliques, T1 plants from 58 (ABCG9 ${ }_{\text {pro: }}$ GUS) and 23 (ABCG31 $1_{\text {pro: }}$ GUS) independent transgenic lines were stained with GUS staining solution as described by Kim et al. (2009). In the case of $A B C G 9$, most lines exhibited similar expression patterns. In $A B C G 31,18$ lines displayed similar expression patterns, and the remaining lines had extended but nonspecific expression. To observe GUS expression in young seedlings, T2 plants from 18 independent 9-d-old transgenic lines each for ABCG9 and ABCG31 were examined after staining with GUS solution. All $A B C G 9$ lines displayed similar expression patterns (no signal). ABCG31 lines varied in the levels and patterns of GUS expression, but 11 of these lines showed similar patterns in shoots and 10 in roots. Flowers and seedlings were incubated in GUS solution for 15 and $12 \mathrm{~h}$, respectively. To observe GUS expression in specific floral tissues, GUS-stained flower buds were fixed and dehydrated as described by Kim et al. (2009). The dehydrated samples were embedded in Technovit 7100 (Kulzer), and then serial sections (10- $\mu \mathrm{m}$ thick) were obtained using a Leica RM2245 rotary microtome. Sections were observed using a Zeiss Axioskop2 microscope (Carl Zeiss).

For detailed observation of the GUS signal driven by the ABCG9 promoter in the flower and silique, GUS-stained flowers and siliques from transgenic lines expressing $A B C G 9_{\text {pro }}$ GUS were cleared in chloral hydrate (chloral hydrate:water:glycerol, $8: 2: 1, w / v / v)$. Cleared flowers and siliques were observed using a Zeiss Axioskop2 microscope. Individual developing seeds were separated from the silique using a syringe needle before observation.

\section{qRT-PCR Analysis}

Total RNA was extracted from 2-week-old seedlings and from different tissues in mature wild-type plants. cDNA was synthesized from isolated RNAs by reverse transcription. qRT-PCR was performed using the SYBR 
kit (Takara), following the manufacturer's instructions. The relative abundance of cDNA was normalized against that of PP2A (Czechowski et al., 2005).

\section{Overexpressing or Complementation Constructs}

ABCG9- or ABCG31-overexpressing constructs (35Spro:sGFP:ABCG9 or 35Spro:sGFP:ABCG31) and the ABCG9 complementation construct (ABCG9pro:sGFP:ABCG9) were generated. Construct information is available in Supplemental Table 3.

ABCG9 complementation lines were generated using the Agrobacteriummediated floral dipping method and then selected by growth on half-strength Murashige and Skoog medium containing $80 \mu \mathrm{g} / \mathrm{mL}$ gentamycin in the T1 generation and by antibiotic resistance and GFP fluorescence in the anthers in the T2 generation. One multiple-insertion line (Compl-1) and two singleinsertion lines (Compl-2 and Compl-3) were chosen for the complementation assays. Each line was grown on antibiotic-containing medium, and the surviving plants were used for the assays.

\section{Subcellular Localization of ABCG9 and ABCG31 in Nicotiana benthamiana Leaves}

To investigate the subcellular localization of ABCG9 and ABCG31 in plant cells, Agrobacterium strain GV3101, which was transformed with 35Spro: sGFP:ABCG9 or 35Spro:sGFP:ABCG31 or anti-silencing vector RK19 to reduce the silencing of the transgenes, was grown in yeast extractpeptone liquid medium overnight. The culture was centrifuged at $1769 \mathrm{~g}$ for $15 \mathrm{~min}$ and resuspended in AS medium (10 mM MgCl $2,10 \mathrm{mM}$ MES$\mathrm{KOH}, \mathrm{pH} 5.6$, and $150 \mu \mathrm{M}$ acetosyringone) by adjusting the OD to 0.7 to 0.8 at $600 \mathrm{~nm}$. The RK19 clone was mixed with the ABCG9 or ABCG31 clone at a ratio of $1: 1$ to a final volume of $1 \mathrm{~mL}$ and incubated for 2 to $4 \mathrm{~h}$. Well-watered 2- to 4-week-old $N$. benthamiana leaves were inoculated with each mixture using a syringe.

Sixty-two hours after inoculation, the fluorescence was tested in transgenic $N$. benthamiana leaf discs. Then, the protoplasts were isolated by enzyme treatment and observed using a Zeiss Axioskop2 fluorescence microscope. GFP fluorescence was observed at 504 to $534 \mathrm{~nm}$ for emission and 465 to $485 \mathrm{~nm}$ for excitation, and chlorophyll autofluorescence was detected at 575 to $640 \mathrm{~nm}$ for emission and at $546 / 12 \mathrm{~nm}$ for excitation. Images obtained at the two spectral settings were overlaid.

\section{Subcellular Localization of ABCG9 in the Anther}

Flowers of the Compl-1 line were removed at various stages. The anthers were isolated from each flower using needles and then observed using an FV1000 confocal laser scanning microscope (Olympus) with spectral settings for GFP (excitation, $488 \mathrm{~nm}$; emission, $520 \mathrm{~nm}$ ). To visualize the anther cells, chlorophyll autofluorescence was observed at $543 \mathrm{~nm}$ for excitation and 613 $\mathrm{nm}$ for emission. Images obtained at the two spectral settings were overlaid.

\section{Pollen Viability Test}

Pollen of freshly opened flowers from 4- to 5 -week-old plants was incubated in a solution containing $0.5 \mu \mathrm{g} / \mathrm{mL}$ FDA and $2 \mu \mathrm{g} / \mathrm{mL}$ PPI for $5 \mathrm{~min}$, and fluorescence images of the pollen were captured using a Zeiss Axioskop2 fluorescence microscope equipped with a cooled chargecoupled device camera (Carl Zeiss). The spectral settings of the microscope were 504 to $534 \mathrm{~nm}$ for emission and 465 to $485 \mathrm{~nm}$ for excitation to detect the FDA green fluorescence and 575 to $640 \mathrm{~nm}$ for emission and $546 / 12 \mathrm{~nm}$ for excitation to detect the PPI red fluorescence. The number of living (stained only by FDA) and dead (stained only by PPI) pollen grains was counted separately. In the pollen viability assay, the number of living pollen was presented as a percentage of the total number of pollen (i.e., the sum of the number of living and dead pollen).
For the pollen viability assay under high humidity, 4-week-old plants were incubated in a humidity-controlled growth room with $70 \%$ humidity for the remainder of the assay. For the pollen viability assay after cold shock, 4-week-old plants were transferred to a $4^{\circ} \mathrm{C}$ growth chamber, incubated for $3 \mathrm{~d}$, and then brought back to $22^{\circ} \mathrm{C}$. After a 3-d recovery period, pollen viability was examined.

\section{Seed Yield Test}

To determine seed yield after cold shock and under normal conditions, 4-week-old plants were transferred to a $4.5^{\circ} \mathrm{C}$ growth chamber or kept at $22^{\circ} \mathrm{C}$ after removal of elongated siliques. For cold treatment, the plants were incubated at $4.5^{\circ} \mathrm{C}$ for $7 \mathrm{~d}$ and then brought back to $22^{\circ} \mathrm{C}$. After 3 weeks, the seed number of three (cold shock) or four (normal condition) siliques for each plant was counted and averaged.

\section{Observation of Pollen Development}

To compare pollen development in wild-type and abcg9 abcg31 plants, floral buds were prepared at various developmental stages and embedded in Spurr's resin as described by Choi et al. (2011). Serial sections (2 $\mu \mathrm{m}$ thick) were obtained using a Leica RM2265 rotary microtome. Sections were mounted and observed as described by Choi et al. (2011).

\section{Observation of Pollen and the Tapetum Using Electron Microscopy}

To observe the pollen surface using scanning electron microscopy, the pollen of freshly opened flowers, immediately after anthesis, was applied directly to a sticky stub without any fixation. The pollen was sputter-coated with palladium and then observed using a LEO1450VP scanning electron microscope (Carl Zeiss). To observe the pollen coat using TEM, flowers just before anthesis were embedded in Spurr's resin and processed as described above.

To observe the accumulation of lipidic suborganelles in the tapetum using TEM, flowers at the stage just prior to tapetal degeneration were embedded in Spurr's resin and processed as described above.

\section{Pollen Collection and Lipid Analysis}

Mature pollen was collected using the vacuum method (JohnsonBrousseau and McCormick, 2004) from more than 3000 plants each for the wild type, abcg9-1, abcg31-1, and abcg9 abcg31, transferred into a glass vial, and freeze-dried. For sterol analysis, freeze-dried pollen was washed three times with $2 \mathrm{~mL}$ of $\mathrm{CHCl}_{3}$ :methanol (1:1). After the addition of Celite to the extract, the solvents were removed under reduced pressure and the extracts were adsorbed onto Celite. The adsorbed samples were placed in a Sep-Pak Vac (silica, $2 \mathrm{~g} / 12 \mathrm{cc}$; Waters) and eluted with $10 \mathrm{~mL}$ of hexane: $\mathrm{CHCl}_{3}(1: 3)$, hexane:EtOAc (2:1), and $\mathrm{CHCl}_{3}$ : methanol (1:1), and $\left[25,26,26,26,27,27,27-{ }^{2} \mathrm{H}_{7}\right]$ cholesterol $\left(98 \%{ }^{2} \mathrm{H}\right.$; Cambridge Isotope Laboratories) was added to the extracts as an internal standard. The hexane: $\mathrm{CHCl}_{3}$ eluent (esterified sterol fraction) was dried and saponified with $1 \mathrm{~mL}$ of $4 \mathrm{M} \mathrm{KOH}$ in ethanol for $1 \mathrm{~h}$ at $80^{\circ} \mathrm{C}$, and then $1 \mathrm{~mL}$ of water was added. The $\mathrm{CHCl}_{3}$ :methanol eluent (steryl glycoside fraction) was dried and hydrolyzed with $1 \mathrm{~mL}$ each of methanol and $4 \mathrm{M}$ $\mathrm{HCl}$ for $1 \mathrm{~h}$ at $80^{\circ} \mathrm{C}$. These reaction mixtures were then extracted three times with $2 \mathrm{~mL}$ of hexane, and each hexane layer was evaporated to dryness. The hexane:ethyl acetate eluent (free sterol fraction) was dried in vacuo. These three residues were trimethylsilylated and analyzed by gas chromatography-mass spectrometry (GC-MS). The endogenous levels of sterols were determined as the peak area ratios of molecular ions in the endogenous residue to those in the internal standard. GC-MS analyses were performed under the following conditions. A mass spectrometer (JMS-AM SUN200; JEOL) was connected to a gas chromatograph (6890A; Agilent Technologies) at an electron ionization of $70 \mathrm{eV}$, source 
temperature of $250^{\circ} \mathrm{C}$, DB- 1 column of $30 \mathrm{~m} \times 0.25 \mathrm{~mm}$, film thickness of $0.25 \mu \mathrm{m}$ (J\&W Scientific), and an injection temperature of $250^{\circ} \mathrm{C}$, and the following column temperature program was used: $80^{\circ} \mathrm{C}$ for $1 \mathrm{~min}$, raised to $280^{\circ} \mathrm{C}$ at a rate of $95^{\circ} \mathrm{C} / \mathrm{min}$, and held at $280^{\circ} \mathrm{C}$ for $12 \mathrm{~min}$. The interface temperature was $280^{\circ} \mathrm{C}$, the carrier gas was helium, and the flow rate was $1 \mathrm{~mL} / \mathrm{min}$. Splitless injection was performed. Samples were trimethylsilylated with $N$-methyl- $N$-trimethylsilyltrifluoroacetamide (Sigma-Aldrich) at $80^{\circ} \mathrm{C}$ for $30 \mathrm{~min}$.

The pollen wax analysis was performed with some modifications from that of Preuss et al. (1993) and Fiebig et al. (2000). Ten milligrams of freeze-dried pollen grains was transferred into a glass tube, and hexane and internal standards, $n$-octacosane, docosanoic acid, and 1-tricosanol, were added. The tube containing pollen grains was shaken at $5^{\circ} \mathrm{C}$ for $2 \mathrm{~h}$, and then pollen wax was extracted. The solvent was removed under a gentle stream of nitrogen gas. The remaining wax mixtures were incubated with bis- $N, N$-(trimethylsilyl)trifluoroacetamide (Sigma-Aldrich) in pyridine for $30 \mathrm{~min}$ at $90^{\circ} \mathrm{C}$ to transform the waxes into trimethylsilyl derivatives. The qualitative and quantitative analyses were performed using GC-MS (GCMS-QP2010; Shimazu) and gas chromatography (GC2010; Shimazu) with an HP-5 column of $60 \mathrm{~m} \times 0.32 \mathrm{~mm}$ and film thickness of $0.25 \mathrm{~mm}$ (Agilent). The following column temperature program was used: $220^{\circ} \mathrm{C}$ for $4.5 \mathrm{~min}$, increased to $290^{\circ} \mathrm{C}$ at a rate of $2^{\circ} \mathrm{C} /$ $\mathrm{min}$, and held at $290^{\circ} \mathrm{C}$ for $15 \mathrm{~min}$. Each compound was quantified against the internal standard by automatically integrating the peak areas.

For brassinosteroid analysis, freeze-dried pollen was washed twice with $100 \mathrm{~mL}$ of methanol: $\mathrm{CHCl}_{3}$ (4:1). Deuterium-labeled internal standards were added to the extracts. Purification and quantification of brassinosteroids were performed according to a previously described method (Fujioka et al., 2002). Pollen from the same plants was used for the analyses of wax, brassinosteroid, and sterol contents.

\section{Primers Used in This Study}

Primer information is available in Supplemental Table 2.

\section{Accession Numbers}

Sequence data from this article can be found in the GenBank/EMBL data libraries under the following accession numbers: ABCG9 (AT4G27420), ABCG31 (AT2G29940), UGT80A2 (AT3G07020), and UGT80B1 (AT1G43620).

\section{Supplemental Data}

The following materials are available in the online version of this article.

Supplemental Figure 1. Expression Patterns of ABCG9 and ABCG31 in Flowers and Developing Seeds, According to the BAR Arabidopsis eFP Browser.

Supplemental Figure 2. Isolation of abcg9 Knockout Mutant Plants. Supplemental Figure 3. Isolation of abcg31 Knockout Mutant Plants.

Supplemental Figure 4. Isolation of abcg9 abcg31 Double Knockout Mutant Plants.

Supplemental Figure 5. Reduced Pollen Viability of abcg9-1 abcg31-2 Plants.

Supplemental Figure 6. Reduced Pollen Viability of abcg9 Plants after Cold Shock.

Supplemental Figure 7. Pattern of $A B C G 9$ and $A B C G 31$ Expression in Tissues Other Than Flowers.

Supplemental Figure 8. Normal Pollen Development in abcg9-1 abcg31-1 Plants.

Supplemental Figure 9. Wild-Type Pollen Grains at the Beginning of Pollen Coat Deposition.
Supplemental Figure 10. Free Sterol and Steryl Ester Contents in Wild-Type, abcg9-1, abcg31-1, and abcg9-1 abcg31-1 Pollen.

Supplemental Figure 11. Identification of MW470 Sterol Compounds.

Supplemental Figure 12. Wax Content in Wild-Type and abcg9-1 abcg31-1 Pollen.

Supplemental Table 1. Brassinosteroid Content in Wild-Type and abcg9-1 abcg31-1 Pollen.

Supplemental Table 2. Primers Used in This Study.

Supplemental Table 3. Constructs.

Supplemental Methods. Comparison of the Steryl Glycoside Content on the Surface of Individual Pollen and Leaf Cells.

Supplemental Data Set 1. Stamen-Specific Expression of ABCG9 and $A B C G 31$ from the MPSS Plus Database.

Supplemental Data Set 2. Genes Coexpressed with ABCG9 or ABCG31.

\section{ACKNOWLEDGMENTS}

We thank Suguru Takatsuto (Joetsu University of Education) for supplying deuterium-labeled internal standards for brassinosteroid analysis, Seth DeBolt (Department of Horticulture, University of Kentucky) for kindly providing ugt80A2 ugt80B1 seeds, and Enrico Martinoia (Institute of Plant Biology, University of Zürich) for helpful discussions. This work was supported by the Global Frontier Program (Grant 2012055051), the Global Research Laboratory program funded by the Ministry of Science, Information and Communication Technology, and Future Planning (Grant R31-2008-000-10105-0), and the Biogreen21 Program of the Rural Development Administration (Grant PJ009484 to Y.L.).

\section{AUTHOR CONTRIBUTIONS}

H.C., Y.-Y.K., and Y.L. designed the research. H.C., K.O., J.-Y.J., S.B.L., S.F., and Y.Y. performed the experiments. All authors analyzed the data. H.C. and Y.L. wrote the article.

Received October 2, 2013; revised December 11, 2013; accepted January 9, 2014; published January 28, 2014.

\section{REFERENCES}

Aarts, M.G., Hodge, R., Kalantidis, K., Florack, D., Wilson, Z.A., Mulligan, B.J., Stiekema, W.J., Scott, R., and Pereira, A. (1997). The Arabidopsis MALE STERILITY 2 protein shares similarity with reductases in elongation/condensation complexes. Plant J. 12: 615-623.

Berge, K.E., Tian, H., Graf, G.A., Yu, L., Grishin, N.V., Schultz, J., Kwiterovich, P., Shan, B., Barnes, R., and Hobbs, H.H. (2000). Accumulation of dietary cholesterol in sitosterolemia caused by mutations in adjacent ABC transporters. Science 290: 1771-1775.

Bessire, M., Borel, S., Fabre, G., Carraça, L., Efremova, N., Yephremov, A., Cao, Y., Jetter, R., Jacquat, A.C., Métraux, J.P., and Nawrath, C. (2011). A member of the PLEIOTROPIC DRUG RESISTANCE family of ATP binding cassette transporters is required for the formation of a functional cuticle in Arabidopsis. Plant Cell 23: 1958-1970.

Bienert, M.D., Siegmund, S.E., Drozak, A., Trombik, T., Bultreys, A., Baldwin, I.T., and Boutry, M. (2012). A pleiotropic drug 
resistance transporter in Nicotiana tabacum is involved in defense against the herbivore Manduca sexta. Plant J. 72: 745-757.

Bird, D., Beisson, F., Brigham, A., Shin, J., Greer, S., Jetter, R., Kunst, L., Wu, X., Yephremov, A., and Samuels, L. (2007). Characterization of Arabidopsis ABCG11/WBC11, an ATP binding cassette $(A B C)$ transporter that is required for cuticular lipid secretion. Plant J. 52: 485-498.

Bowman, J.L., Smyth, D.R., and Meyerowitz, E.M. (1989). Genes directing flower development in Arabidopsis. Plant Cell 1: 37-52.

Choi, H., Jin, J.Y., Choi, S., Hwang, J.U., Kim, Y.Y., Suh, M.C., and Lee, Y. (2011). An ABCG/WBC-type ABC transporter is essential for transport of sporopollenin precursors for exine formation in developing pollen. Plant J. 65: 181-193.

Clough, S.J., and Bent, A.F. (1998). Floral dip: A simplified method for Agrobacterium-mediated transformation of Arabidopsis thaliana. Plant J. 16: 735-743.

Crouzet, J., Roland, J., Peeters, E., Trombik, T., Ducos, E., Nader, J., and Boutry, M. (2013). NtPDR1, a plasma membrane ABC transporter from Nicotiana tabacum, is involved in diterpene transport. Plant Mol. Biol. 82: 181-192.

Czechowski, T., Stitt, M., Altmann, T., Udvardi, M.K., and Scheible, W.R. (2005). Genome-wide identification and testing of superior reference genes for transcript normalization in Arabidopsis. Plant Physiol. 139: 5-17.

de Azevedo Souza, C., Kim, S.S., Koch, S., Kienow, L., Schneider, K., McKim, S.M., Haughn, G.W., Kombrink, E., and Douglas, C.J. (2009). A novel fatty acyl-CoA synthetase is required for pollen development and sporopollenin biosynthesis in Arabidopsis. Plant Cell 21: 507-525.

DeBolt, S., et al. (2009). Mutations in UDP-glucose:sterol glucosyltransferase in Arabidopsis cause transparent testa phenotype and suberization defect in seeds. Plant Physiol. 151: 78-87.

Demchik, S.M., and Day, T.A. (1996). Effect of enhanced UV-B radiation on pollen quantity, quality, and seed yield in Brassica rapa (Brassicaceae). Am. J. Bot. 83: 573-579.

Dobritsa, A.A., Lei, Z., Nishikawa, S., Urbanczyk-Wochniak, E., Huhman, D.V., Preuss, D., and Sumner, L.W. (2010). LAP5 and $\angle A P 6$ encode anther-specific proteins with similarity to chalcone synthase essential for pollen exine development in Arabidopsis. Plant Physiol. 153: 937-955.

Dobritsa, A.A., Shrestha, J., Morant, M., Pinot, F., Matsuno, M., Swanson, R., Møller, B.L., and Preuss, D. (2009). CYP704B1 is a long-chain fatty acid $\omega$-hydroxylase essential for sporopollenin synthesis in pollen of Arabidopsis. Plant Physiol. 151: 574-589.

Dou, X.Y., Yang, K.Z., Zhang, Y., Wang, W., Liu, X.L., Chen, L.Q., Zhang, X.Q., and Ye, D. (2011). WBC27, an adenosine triphosphate-binding cassette protein, controls pollen wall formation and patterning in Arabidopsis. J. Integr. Plant Biol. 53: 74-88.

Fiebig, A., Mayfield, J.A., Miley, N.L., Chau, S., Fischer, R.L., and Preuss, D. (2000). Alterations in CER6, a gene identical to CUT1, differentially affect long-chain lipid content on the surface of pollen and stems. Plant Cell 12: 2001-2008.

Francia, P., Simoni, L., Cominelli, E., Tonelli, C., and Galbiati, M. (2008). Gene trap-based identification of a guard cell promoter in Arabidopsis. Plant Signal. Behav. 3: 684-686.

Fujioka, S., Takatsuto, S., and Yoshida, S. (2002). An early C-22 oxidation branch in the brassinosteroid biosynthetic pathway. Plant Physiol. 130: 930-939.

Geisler, M., et al. (2005). Cellular efflux of auxin catalyzed by the Arabidopsis MDR/PGP transporter AtPGP1. Plant J. 44: 179-194.

Goldberg, R.B., Beals, T.P., and Sanders, P.M. (1993). Anther development: Basic principles and practical applications. Plant Cell 5: 1217-1229.
Grove, M.D., Spencer, G.F., Rohwedder, W.K., Mandava, N., Worley, J.F., Warthen, J.D., Steffens, G.L., Flippen-Anderson, J.L., and Cook, J.C. (1979). Brassinolide, a plant growth-promoting steroid isolated from Brassica napus pollen. Nature 281: 216-217.

Hartmann, M.A., and Benveniste, P. (1987). Plant membrane sterols: Isolation, identification, and biosynthesis. Methods Enzymol. 148: 632-650.

Hsieh, K., and Huang, A.H. (2005). Lipid-rich tapetosomes in Brassica tapetum are composed of oleosin-coated oil droplets and vesicles, both assembled in and then detached from the endoplasmic reticulum. Plant J. 43: 889-899.

Hsieh, K., and Huang, A.H. (2007). Tapetosomes in Brassica tapetum accumulate endoplasmic reticulum-derived flavonoids and alkanes for delivery to the pollen surface. Plant Cell 19: 582-596.

Janvilisri, T., Venter, H., Shahi, S., Reuter, G., Balakrishnan, L., and van Veen, H.W. (2003). Sterol transport by the human breast cancer resistance protein (ABCG2) expressed in Lactococcus lactis. J. Biol. Chem. 278: 20645-20651.

Jessen, D., Olbrich, A., Knüfer, J., Krüger, A., Hoppert, M., Polle, A., and Fulda, M. (2011). Combined activity of LACS1 and LACS4 is required for proper pollen coat formation in Arabidopsis. Plant J. 68: 715-726.

Johnson-Brousseau, S.A., and McCormick, S. (2004). A compendium of methods useful for characterizing Arabidopsis pollen mutants and gametophytically-expressed genes. Plant J. 39: 761-775.

Kang, J., Park, J., Choi, H., Burla, B., Kretzschmar, T., Lee, Y., and Martinoia, E. (2011). Plant ABC transporters. The Arabidopsis Book 9: e0153, doi/10.1199/tab.0153.

Kim, Y.Y., Choi, H., Segami, S., Cho, H.T., Martinoia, E., Maeshima, M., and Lee, Y. (2009). AtHMA1 contributes to the detoxification of excess $\mathrm{Zn}(\mathrm{II})$ in Arabidopsis. Plant J. 58: 737-753.

Kuromori, T., Sugimoto, E., and Shinozaki, K. (2011). Arabidopsis mutants of $A t A B C G 22$, an $A B C$ transporter gene, increase water transpiration and drought susceptibility. Plant J. 67: 885-894.

Lansac, A.R., Sullivan, C.Y., Johnson, B.E., and Lee, K.W. (1994). Viability and germination of the pollen of sorghum [Sorghum bicolor (L.) Moench]. Ann. Bot. (Lond.) 74: 27-33.

Lee, J.Y., and Lee, D.H. (2003). Use of serial analysis of gene expression technology to reveal changes in gene expression in Arabidopsis pollen undergoing cold stress. Plant Physiol. 132: 517-529.

Le Hir, R., Sorin, C., Chakraborti, D., Moritz, T., Schaller, H., Tellier, F., Robert, S., Morin, H., Bako, L., and Bellini, C. (2013). ABCG9, ABCG11 and ABCG14 ABC transporters are required for vascular development in Arabidopsis. Plant J. 76: 811-824.

Li, Y., and Prinz, W.A. (2004). ATP-binding cassette (ABC) transporters mediate nonvesicular, raft-modulated sterol movement from the plasma membrane to the endoplasmic reticulum. J. Biol. Chem. 279: 4522645234.

Luo, B., Xue, X.Y., Hu, W.L., Wang, L.J., and Chen, X.Y. (2007). An ABC transporter gene of Arabidopsis thaliana, AtWBC11, is involved in cuticle development and prevention of organ fusion. Plant Cell Physiol. 48: 1790-1802.

Manfield, I.W., Jen, C.H., Pinney, J.W., Michalopoulos, I., Bradford, J.R., Gilmartin, P.M., and Westhead, D.R. (2006). Arabidopsis Coexpression Tool (ACT): Web server tools for microarray-based gene expression analysis. Nucleic Acids Res. 34: W504-W509.

Matsuda, N., Tsuchiya, T., Kishitani, S., Tanaka, Y., and Toriyama, K. (1996). Partial male sterility in transgenic tobacco carrying antisense and sense PAL cDNA under the control of a tapetum-specific promoter. Plant Cell Physiol. 37: 215-222.

Morant, M., Jørgensen, K., Schaller, H., Pinot, F., Møller, B.L., WerckReichhart, D., and Bak, S. (2007). CYP703 is an ancient cytochrome P450 in land plants catalyzing in-chain hydroxylation of lauric acid to 
provide building blocks for sporopollenin synthesis in pollen. Plant Cell 19: 1473-1487.

Oku, M., Warnecke, D., Noda, T., Müller, F., Heinz, E., Mukaiyama, H., Kato, N., and Sakai, Y. (2003). Peroxisome degradation requires catalytically active sterol glucosyltransferase with a GRAM domain. EMBO J. 22: 3231-3241.

Pacini, E., and Franchi, G.G. (1993). Role of the tapetum in pollen and spore dispersal. Plant Syst. Evol. 7: 1-11.

Palta, J.P., Whitaker, B.D., and Weiss, L.S. (1993). Plasma membrane lipids associated with genetic variability in freezing tolerance and cold acclimation of Solanum species. Plant Physiol. 103: 793-803.

Panikashvili, D., Shi, J.X., Schreiber, L., and Aharoni, A. (2011). The Arabidopsis ABCG13 transporter is required for flower cuticle secretion and patterning of the petal epidermis. New Phytol. 190: 113-124.

Panikashvili, D., Savaldi-Goldstein, S., Mandel, T., Yifhar, T., Franke, R.B., Höfer, R., Schreiber, L., Chory, J., and Aharoni, A. (2007). The Arabidopsis DESPERADO/AtWBC11 transporter is required for cutin and wax secretion. Plant Physiol. 145: 1345-1360.

Patkar, R.N., Xue, Y.K., Shui, G., Wenk, M.R., and Naqvi, N.I. (2012). Abc3-mediated efflux of an endogenous digoxin-like steroidal glycoside by Magnaporthe oryzae is necessary for host invasion during blast disease. PLoS Pathog. 8: e1002888.

Peng, L., Kawagoe, Y., Hogan, P., and Delmer, D. (2002). Sitosterolbeta-glucoside as primer for cellulose synthesis in plants. Science 295: $147-150$

Pickert, M. (1988). In vitro germination and storage of trinucleate Arabidopsis thaliana (L.) pollen grains. Arabidopsis Inf. Service 26: 39-42.

Piffanelli, P., Ross, J.H., and Murphy, D.J. (1998). Biogenesis and function of the lipidic structures of pollen grains. Sex. Plant Reprod. 11: $65-80$.

Pighin, J.A., Zheng, H., Balakshin, L.J., Goodman, I.P., Western, T.L., Jetter, R., Kunst, L., and Samuels, A.L. (2004). Plant cuticular lipid export requires an ABC transporter. Science 306: 702-704.

Preuss, D., Lemieux, B., Yen, G., and Davis, R.W. (1993). A conditional sterile mutation eliminates surface components from Arabidopsis pollen and disrupts cell signaling during fertilization. Genes Dev. 7: 974-985

Quilichini, T.D., Friedmann, M.C., Samuels, A.L., and Douglas, C.J. (2010). ATP-binding cassette transporter G26 is required for male fertility and pollen exine formation in Arabidopsis. Plant Physiol. 154: 678-690.

Rao, G.U., Jain, A., and Shivanna, K.R. (1992). Effects of high temperature stress on Brassica pollen: Viability, germination and ability to set fruits and seeds. Ann. Bot. (Lond.) 69: 193-198.

Rea, P.A. (2007). Plant ATP-binding cassette transporters. Annu. Rev. Plant Biol. 58: 347-375.

Regan, S.M., and Moffatt, B.A. (1990). Cytochemical analysis of pollen development in wild-type Arabidopsis and a male-sterile mutant. Plant Cell 2: 877-889.

Ruzicka, K., et al. (2010). Arabidopsis PIS1 encodes the ABCG37 transporter of auxinic compounds including the auxin precursor indole3-butyric acid. Proc. Natl. Acad. Sci. USA 107: 10749-10753.

Sakai, H., Krizek, B.A., Jacobsen, S.E., and Meyerowitz, E.M. (2000). Regulation of SUP expression identifies multiple regulators involved in Arabidopsis floral meristem development. Plant Cell 12: 1607-1618.
Scott, R.J., Spielman, M., and Dickinson, H.G. (2004). Stamen structure and function. Plant Cell 16 (suppl.): S46-S60.

Stieglitz, H. (1977). Role of $\beta-1,3-$ glucanase in postmeiotic microspore release. Dev. Biol. 57: 87-97.

Strader, L.C., and Bartel, B. (2009). The Arabidopsis PLEIOTROPIC DRUG RESISTANCE8/ABCG36 ATP binding cassette transporter modulates sensitivity to the auxin precursor indole-3-butyric acid. Plant Cell 21: 1992-2007.

Tanigawara, Y., Okamura, N., Hirai, M., Yasuhara, M., Ueda, K., Kioka, N., Komano, T., and Hori, R. (1992). Transport of digoxin by human P-glycoprotein expressed in a porcine kidney epithelial cell line (LLC-PK1). J. Pharmacol. Exp. Ther. 263: 840-845.

Terasaka, K., Blakeslee, J.J., Titapiwatanakun, B., Peer, W.A., Bandyopadhyay, A., Makam, S.N., Lee, O.R., Richards, E.L., Murphy, A.S., Sato, F., and Yazaki, K. (2005). PGP4, an ATP binding cassette P-glycoprotein, catalyzes auxin transport in Arabidopsis thaliana roots. Plant Cell 17: 2922-2939.

Ukitsu, H., et al. (2007). Cytological and biochemical analysis of COF1, an Arabidopsis mutant of an ABC transporter gene. Plant Cell Physiol. 48: 1524-1533.

Ullmann, P., Bouvier-Navé, P., and Benveniste, P. (1987). Regulation by phospholipids and kinetic studies of plant membrane-bound UDPglucose sterol $\beta$-D-glucosyl transferase. Plant Physiol. 85: 51-55.

van der Meer, I.M., Stam, M.E., van Tunen, A.J., Mol, J.N., and Stuitje, A.R. (1992). Antisense inhibition of flavonoid biosynthesis in petunia anthers results in male sterility. Plant Cell 4: 253-262.

Velamakanni, S., Wei, S.L., Janvilisri, T., and van Veen, H.W. (2007). ABCG transporters: Structure, substrate specificities and physiological roles. A brief overview. J. Bioenerg. Biomembr. 39: 465-471.

Verrier, P.J., et al. (2008). Plant ABC proteins-A unified nomenclature and updated inventory. Trends Plant Sci. 13: 151-159.

Wang, J., Zhang, D.W., Lei, Y., Xu, F., Cohen, J.C., Hobbs, H.H., and Xie, X.S. (2008a). Purification and reconstitution of sterol transfer by native mouse ABCG5 and ABCG8. Biochemistry 47: 5194-5204.

Wang, N., Yvan-Charvet, L., Lütjohann, D., Mulder, M., Vanmierlo, T., Kim, T.W., and Tall, A.R. (2008b). ATP-binding cassette transporters $\mathrm{G} 1$ and $\mathrm{G} 4$ mediate cholesterol and desmosterol efflux to $\mathrm{HDL}$ and regulate sterol accumulation in the brain. FASEB J. 22: 1073-1082.

Warnecke, D., Erdmann, R., Fahl, A., Hube, B., Müller, F., Zank, T., Zähringer, U., and Heinz, E. (1999). Cloning and functional expression of UGT genes encoding sterol glucosyltransferases from Saccharomyces cerevisiae, Candida albicans, Pichia pastoris, and Dictyostelium discoideum. J. Biol. Chem. 274: 13048-13059.

Wilcox, L.J., Balderes, D.A., Wharton, B., Tinkelenberg, A.H., Rao, G., and Sturley, S.L. (2002). Transcriptional profiling identifies two members of the ATP-binding cassette transporter superfamily required for sterol uptake in yeast. J. Biol. Chem. 277: 32466-32472.

Xi, J., Xu, P., and Xiang, C.B. (2012). Loss of AtPDR11, a plasma membrane-localized $A B C$ transporter, confers paraquat tolerance in Arabidopsis thaliana. Plant J. 69: 782-791.

Xu, J., Yang, C., Yuan, Z., Zhang, D., Gondwe, M.Y., Ding, Z., Liang, W., Zhang, D., and Wilson, Z.A. (2010). The ABORTED MICROSPORES regulatory network is required for postmeiotic male reproductive development in Arabidopsis thaliana. Plant Cell 22: 91-107. 
The Role of Arabidopsis ABCG9 and ABCG31 ATP Binding Cassette Transporters in Pollen Fitness and the Deposition of Steryl Glycosides on the Pollen Coat

Hyunju Choi, Kiyoshi Ohyama, Yu-Young Kim, Jun-Young Jin, Saet Buyl Lee, Yasuyo Yamaoka, Toshiya Muranaka, Mi Chung Suh, Shozo Fujioka and Youngsook Lee

Plant Cell 2014;26;310-324; originally published online January 28, 2014;

DOI 10.1105/tpc.113.118935

This information is current as of July 25, 2019

Supplemental Data

References

Permissions

eTOCs

CiteTrack Alerts

Subscription Information
/content/suppl/2014/01/24/tpc.113.118935.DC1.html

This article cites 72 articles, 35 of which can be accessed free at: /content/26/1/310.full.html\#ref-list-1

https://www.copyright.com/ccc/openurl.do?sid=pd_hw1532298X\&issn=1532298X\&WT.mc_id=pd_hw1532298X

Sign up for eTOCs at:

http://www.plantcell.org/cgi/alerts/ctmain

Sign up for CiteTrack Alerts at:

http://www.plantcell.org/cgi/alerts/ctmain

Subscription Information for The Plant Cell and Plant Physiology is available at: http://www.aspb.org/publications/subscriptions.cfm 